\title{
Review Article \\ Recent Developments on Wireless Sensor Networks Technology for Bridge Health Monitoring
}

\author{
Guang-Dong $\mathrm{Zhou}^{1}$ and Ting-Hua $\mathrm{Yi}^{2,3}$ \\ ${ }^{1}$ College of Civil and Transportation Engineering, Hohai University, Nanjing 210098, China \\ ${ }^{2}$ School of Civil Engineering, Dalian University of Technology, Dalian 116023, China \\ ${ }^{3}$ Key Laboratory of Concrete and Prestressed Concrete Structure, Ministry of Education, \\ Southeast University, Nanjing 210096, China \\ Correspondence should be addressed to Guang-Dong Zhou; zhougd@hhu.edu.cn
}

Received 24 September 2013; Accepted 21 October 2013

Academic Editor: Stathis C. Stiros

Copyright (C) 2013 G.-D. Zhou and T.-H. Yi. This is an open access article distributed under the Creative Commons Attribution License, which permits unrestricted use, distribution, and reproduction in any medium, provided the original work is properly cited.

\begin{abstract}
Structural health monitoring (SHM) systems have shown great potential to sense the responses of a bridge system, diagnose the current structural conditions, predict the expected future performance, provide information for maintenance, and validate design hypotheses. Wireless sensor networks (WSNs) that have the benefits of reducing implementation costs of SHM systems as well as improving data processing efficiency become an attractive alternative to traditional tethered sensor systems. This paper introduces recent technology developments in the field of bridge health monitoring using WSNs. As a special application of WSNs, the requirements and characteristics of WSNs when used for bridge health monitoring are firstly briefly discussed. Then, the state of the art in WSNs-based bridge health monitoring systems is reviewed including wireless sensor, network topology, data processing technology, power management, and time synchronization. Following that, the performance validations and applications of WSNs in bridge health monitoring through scale models and field deployment are presented. Finally, some existing problems and promising research efforts for promoting applications of WSNs technology in bridge health monitoring throughout the world are explored.
\end{abstract}

\section{Introduction}

A large number of bridges have been built to fulfill the requirement of advanced transportation all over the world and represent the key elements in terms of the safety and functionality of the entire highway system. As those bridges age, not only do structures deteriorate naturally, but also their utilization increases as populations grow in size and mobility. This structural degradation can pose major safety hazards, especially on long-span bridges. Between 1989 and 2000, more than 500 bridges are reported to have partially or totally collapsed in the United States due to triggering events (e.g., earthquake or vehicle collision), design and construction error, and undetected structural deterioration (e.g., scour or fatigue) $[1,2]$. The I-35W Mississippi River bridge located in Minneapolis, Minnesota, USA, is one recent example: it failed on August 1, 2007, collapsing to the river and riverbanks beneath, killing 13 people and injuring 145 [3]. As of 2009, an estimated $24 \%$ of the nation's over 600,000 bridges are currently classified as structurally deficient or functionally obsolete ( $12 \%$ structural deficient) by the Federal Highway Administration (FHWA) of the United States [4]. This suggests that accuracy and reliability of bridge inspections are imperative, and advanced quantitative methods to locate the damage are of pressing importance. Regular visual inspection, which is experiential dependence, traffic disturbance, and high cost, does not appear to be adequate to accurately reflect the true performance state of bridge components or the global condition of the entire bridge. In 2001, the FHWA conducted a study which found that $56 \%$ of mediumto-short-span bridges given an average condition rating by visual inspection were improperly assessed [5]. Structural health monitoring (SHM) that aims at monitoring structural behavior in real-time, evaluating structural performance 
under various loads, and identifying structural damage or deterioration is an attractive alternative. It can be adopted for newly constructed bridges and existing bridges to understand structural health condition and prevent catastrophic failure by prior detection. Furthermore, it helps to verify structural design methodology and promote the development of structural analysis theory. Over the past decade, on-structure longterm monitoring systems have been implemented on bridges in Europe, the United States, Canada, Japan, Korea, China, and other countries [6].

A traditional wired SHM system includes three major components: a sensor system, a data processing system (including the data acquisition, transmission, and storage), and a health evaluation system [7]. The data of the sensor system are transmitted through coaxial wires and processed intensively by the data processing system. There are many disadvantages to the wired SHM system, as follows. (1) The first one is high cost. The extremely long communication cables and corresponding protection pipelines for the sensors require a lot of costs, which causes the investment of the SHM system to be prohibitively high. For example, the average installed cost per sensor of the monitoring system on the Bill Emerson Memorial Bridge is a little more than $\$ 15,000$ [8]; cable wiring used in the SHM system of the Yeongjong Bridge accounts for $50 \%$ of the total installation cost [9]. (2) The second disadvantage is low efficiency. The deployment of miles of cables is labor-intensive and timeconsuming, with potentially over $75 \%$ of the installation time attributed to the installation of system wires and cables for long-span bridges $[10,11]$. Centralized data processing makes the data processing system so busy that the efficiency of structural evaluation is low, which induces the real-time monitoring to become a challenge particularly for those sudden occurrences such as earthquakes and explosions. (3) The third one is susceptible disturbance. A wire-based system has more chances for disturbance or failure caused by nature and human beings since it has more independent components, such as data loggers, sensors, and flimsy cables. The long-distance transmission, changes in cable temperatures, and connections between sensors and cables cause sensor data to be hard-to-correct distortions [9]. (4) The fourth disadvantage is inflexibility. The sensor and cable are pier to pier, which means one coaxial cable only services one sensor generally. Adding or changing sensors implies deploying extra cables for data transmission and renewing data managing software. After the wired SHM system is completed, the modifying of the sensor system results in a lot of accessional work.

The new advances of microelectromechanical systems (MEMS) technology, wireless sensing technology, and integrated circuit technology have realized low-cost wireless sensors with onboard computation and wireless communication capabilities. In order to overcome the intrinsical faultiness of the traditional wired SHM system, the WSNs-based SHM system has recently emerged. The WSNs-based SHM system, which is defined by its use of wireless sensor networks (WSNs) to transfer data from sensor to sensor and from sensor to the central data repository, is considered a viable substitute for a wired monitoring system. The WSNs-based
SHM system, which employs the wireless sensors and WSNs, offers the potential for low-cost and reliable SHM. When compared to the traditional wired SHM system, the WSNsbased SHM system has the following advantages. (1) The first one is low cost. Using the WSNs, the wires are eliminated, and the work of cable installation and protection is released. Employing the onboard data conditioner on the wireless sensor makes the abandonment of the expensive independent demodulator possible. So the cost of the WSNs-based SHM system is reduced dramatically. The prototype wireless system proposed by Kim et al. costs about $\$ 600$ per node compared to thousands of dollars for a node with the same functionalities in a traditional wired network [12]. (2) The second advantage is high efficiency. Without deploying complicated cables, the installation of wireless sensors is very easy [13]. Whelan et al. [14] only spent approximately $2 \mathrm{~h}$ and $4 \mathrm{~h}$, respectively, deploying 30 dual-axis wireless accelerometers and 30 wireless strain transducers on a highway bridge, which is impossible for the traditional wired SHM system. The onboard microprocessor of the wireless sensor can facilitate efficient distributed data processing and real-time damage detection $[15,16]$. (3) The third one is reliability. The hardware of the wireless sensor is highly integrated and there are no additional supporting components, such as long cables, signal analyzer, and data memory. The WSNs-based SHM system is not easy to be disturbed by operation environment, and the reliability is improved. (4) The fourth advantage is flexibility. The sensors in the WSNs-based SHM system are organized by wireless transmission, which makes the updating, adding, moving, and replacing of sensors easy after the initial installation. The network can be reorganized quickly without disturbing the original data acquirement operation [9]. In addition, the rapid reduction in physical size and cost of MEMS-based wireless sensors and improvement of WSNs performance enable deployment of dense arrays of sensors to be feasible and economical, so that the quality of SHM can be dramatically improved with rich information which diagnosis algorithms can utilize to detect, locate, and assess structural damage, which is critical for SHM of complicated and large-scale bridges. Bridge health monitoring using a network of wireless sensors is one of the most promising emerging technologies and is seen as the next generation of SHM [17].

After the use of wireless communications in lieu of wires within a structural monitoring system by Straser and Kiremidjian [18] in 1998 as a means of reducing installation costs in large-scale structures, application of WSNs in bridge health monitoring has gained considerable attention. Now, WSNs technology for bridge health monitoring has seen substantial progress through interdisciplinary research efforts to address issues in sensors, networks, and applicationspecific algorithms. In this paper, the distinguished technology development of WSNs for bridge health monitoring is reviewed. Although the wireless sensors and sensor networks for SHM were summarized by Lynch and Loh in 2006 [19], the hardware of the wireless sensor node was emphasized in that paper. More importantly, most of the early attempts simply replaced wired communication with radio frequency (RF) links, which imply many flaws, such 
as unreliable communication, sampling rate fluctuation, and inaccurate time synchronization. The rapid development of WSNs technology in recent years promotes the emergence of many novel and outstanding concepts in WSNs-based bridge health monitoring, which makes the performance of the system improve dramatically. The interest of this paper is the key issues related to the application of WSNs in bridge health monitoring. In Section 2, the requirements and characteristics of WSNs-based bridge health monitoring are discussed. In Section 3, the progress on the WSNs-based bridge health monitoring system involving wireless sensor, network topology, data processing technology, power management, and time synchronization is presented. In Section 4, performance validation and application of wireless sensors and WSNs in bridge health monitoring are given. Finally, some existing problems and promising research efforts in the WSNs-based bridge health monitoring are discussed. It should be noted that this review is not intended to be exhaustive listing but to provide representative examples as references for future researches.

\section{Requirements and Characteristics of WSNs-Based Bridge Health Monitoring}

The applications of WSNs include many fields, like wildlife habits, forest environments, agriculture, health, life rescue, and structural monitoring. When comparing with general applications of WSNs, the WSNs used for bridge health monitoring have some special requirements and characteristics, as follows.

(1) High resolution: the responses of bridges caused by ambient excitation and traffic are very weak generally. The sensor boards used for bridge health monitoring are required to detect such small-amplitude fluctuations of signals. Moreover, the sensor boards should have a low-noise design and should take noise-reducing measurements for weak signal sampling. Commonly, the resolutions of a wireless acceleration sensor and wireless strain sensor should be below $1 \mathrm{mg}$ and $1 \mu \varepsilon$, respectively.

(2) High frequency: most structural assessment methods are based on the dynamic responses of bridges. So, the dynamic test takes up the main part among different bridge monitoring subjects. Since the noise level is usually high in uncontrolled structural environments, oversampling is generally performed to improve the signal-to-noise ratio by reducing the relative noise energy. Therefore, the sampling rate of sensors used for the dynamic test in the bridge health monitoring system should be more than $50 \mathrm{~Hz}$.

(3) High speed: WSN technology enables deployment of dense arrays of sensors, which is critical for increasing the potential of SHM. For the high sampling frequency and the hundreds or thousands of sensors, the tremendous volume of transportation data in WSN is generated and transmitted in each data sensing period. For example, 2-byte data samples collected at $100 \mathrm{~Hz}$ generate $12 \mathrm{kB}$ of data every minute. High speed data transmission is attractive for bridge health monitoring, but the employed low-power radio transceivers now provide a limited bandwidth (e.g., a maximum of 250 kbps in the case of IEEE 802.15.4 networks) [20].
(4) High reliability: because of the noise effect, electromagnetic interference, packet collision, packet headers, and so forth, in a harsh environment like the practical bridge site, high communication reliability to transmit data and disseminate commands without loss of information is a key issue. Data loss would have the effect of increasing the noise, which would make modal identification more difficult and also affect the subsequent condition assessment [21]. Particularly for rare events, data loss is not acceptable.

(5) Long lifetime: bridge health monitoring is a work that runs throughout the whole lifetime of the structure. In general, the service life of a long-span bridge is more than 50 years. The sensors are expected to remain active during those years and have the ability to be interrogated at any time to acquire data. Now, most of the wireless sensors are powered by batteries with limited capacity. So, the implementation of careful power management is one of the most critical features of successful WSN deployment.

(6) Time synchronization: each wireless sensor in WSNs has its own clock, which is not synchronized initially with other sensor nodes. A time synchronization (TS) error of $1 \mathrm{~ms}$ results in an about 3.6-degree phase delay of a mode at $10 \mathrm{~Hz}$, while the same time synchronization error causes a 36degree phase delay at $100 \mathrm{~Hz}$ [21]. The time synchronization error is comparable or can even exceed the effect of sensor's noise [22]. Careful time synchronization is also required in the application of correlation analyses.

(7) Various signal formats: a bridge is a complicate system. To understand the performance of a bridge comprehensively, various parameters such as acceleration, strain, displacement, temperature, and crack need to be monitored. Collecting those parameters, the formats of the signals are heterogeneous. Some are dynamic and the others are static. Some are absolute values and the others are relative values. The WSNs for bridge health monitoring should be flexible to different types of signals.

(8) Long-distance transmission: the total lengths of bridges always exceed the broadcast domain of a node. For example, the main span of the Tsing Ma Bridge is $1377 \mathrm{~m}$, the total length of the Runyang South Bridge is $2430 \mathrm{~m}$, and the total length of the Hangzhou Bay Bridge is 36 miles. Single-hop communication in a large wireless network for bridge health monitoring is impractical. Therefore, multihop wireless communication is demanded [13].

(9) Fixed sensor locations: the aim of WSN deployment is structural condition evaluation. The locations of wireless sensors are selected to fulfill the requirement of structural analysis and damage diagnosis. Once the wireless sensors are arranged, their locations are not changed any more during a measurement period. Therefore, the mobility of wireless sensors is not an issue for bridge health monitoring and there is no need to consider the movement of wireless sensors when developing a WSNs route.

\section{WSNs-Based Bridge Health Monitoring System}

As a new concept in the field of SHM, the WSNs-based bridge health monitoring system, which eliminates the high 


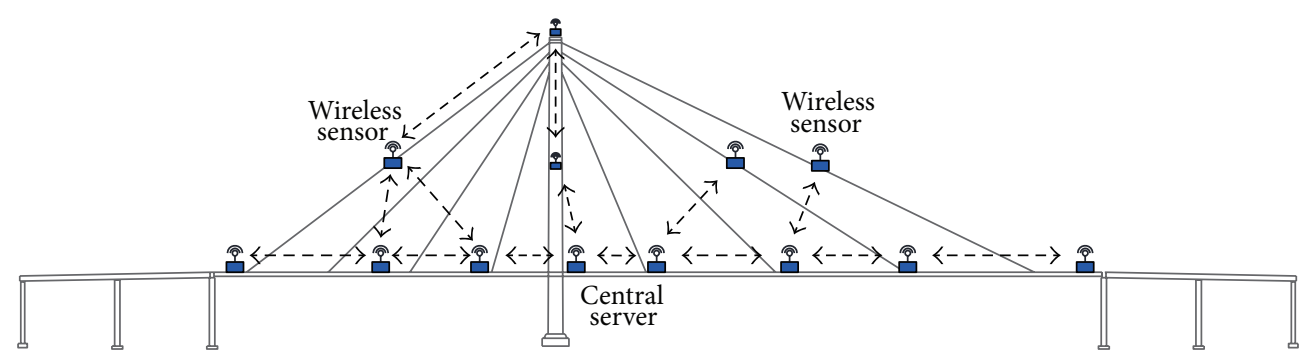

FIGURE 1: WSNs-based bridge health monitoring system.

cost cable, relies on the WSNs to transmit data. A typical WSN for bridge health monitoring is displayed in Figure 1. A lot of wireless sensors are deployed to key locations on a bridge. At first, the central server sends commands to activate wireless sensors, establish a WSN, and set the parameters of monitoring; then, the whole WSN executes TS; after that, the wireless sensors begin collecting data and transmitting raw data or processed results back to the central server; finally, the measured data can be used for advanced structural performance evaluation. Among them, handshake protocols are recommended to ensure the commands or data are sent to the appointed places.

The WSNs-based bridge health monitoring system is comprised of hardware and software. The hardware can be divided into a wireless sensor and central server. Wireless sensors are the cornerstone component of a WSNs-based bridge health monitoring system and are responsible for sensing structural responses and realizing in-network commands. The central server, which includes a gateway connecting to the data repository and the central control system for customized structural evaluation algorithms like that in the wired bridge health monitoring system, executes commands and data sending/receiving. And the software can be divided into different components: network operation, data collecting, data processing, power management, and so forth. The network operation is used for organizing network topology and controlling data stream. The data collecting performs strategies of data collecting such as TS, sampling rate, and sampling period. The data processing carries out onboard data processing, such as data compression, fast Fourier transform (FFT), and power spectrum density (PSD). The power management implements high efficiency power utilization like the sleeping model, triggering measurement, periodical test. Indeed, those components of software overlap, and the realization of an operation may involve different components. In addition, the software development requires an operation system. In this section, the technological development of key issues in the WSNs-based bridge health monitoring system is presented including wireless sensor, network topology, data processing technology, power management, and TS.

3.1. Wireless Sensor. Wireless sensors are not sensors defined by the conventional concept but rather are autonomous data acquisition nodes in which structural sensing elements (e.g., strain gauges, accelerometers, linear voltage displacement transducers, inclinometers, among others), the onboard

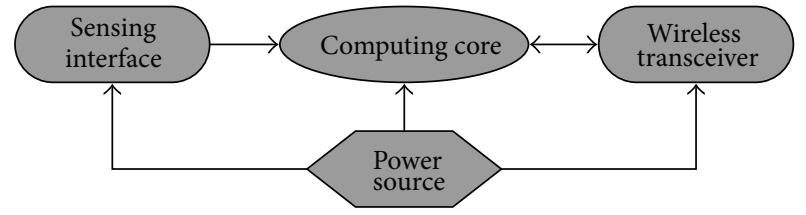

FIGURE 2: Subsystems of a wireless sensor.

microprocessor, and wireless communication elements are integrated. The greatest attribute of the wireless sensor is its collocation of computational resources with the sensor. Such resources can be used for data interrogating, digital signal processing, self-diagnosis, self-calibration, selfidentification, and self-adaptation. This capability is particularly attractive within the context of SHM. So while cost has been an early motivator for considering the installation of wireless sensors in structures, the fact that wireless sensors are a new sensing paradigm offering autonomous data processing is fueling recent excitement. In general, a wireless sensor is composed by four functional subsystems: a sensing interface, a computing core, a wireless transceiver, and a power source, as shown in Figure $2[2,19]$. For some of them, the actuation interface is also integrated, which is beyond the scope of this paper. The sensing interface includes an interface to which sensors can be connected and an analog-to-digital converter (ADC) to convert analog sensor signals to digital formats. The computing core generally consists of a microcontroller for the computational tasks, a random access memory (RAM) to stack the measured and processed data, and a flash memory with software programs for the system operation and data processing. The wireless transceiver is an integral component of the wireless system, which is composed of an RF radio modem and antenna to exchange information with other wireless sensors and to transfer data to the central server [23]. The power source, which is responsible for providing stable energy to the sensing interface, computing core, and wireless transceiver, commonly employs batteries.

The successful utilization of WSNs for bridge monitoring relies on their ability to capture data that provides a reasonable representation of the physical response. However, some of the inherent characteristics associated with wireless sensors have made high-fidelity data acquisition a challenging undertaking. Therefore, to date, a major effort in the field of wireless-based SHM is contributed to the development of high-performance wireless sensors. When designing an applicative wireless sensor for bridge health monitoring, 
the performance and functionality of each subsystem must be carefully selected considering the structural type, quantities to monitor, sensor locations, and environment of the structure [23]. Although there are many commercially available wireless sensor nodes or sensor boards for measuring vibration, their performance cannot meet the requirement of bridge health monitoring mentioned in Section 2. For example, the Basic Sensor Board (ITS400) provided by MEMSIC lacks flexibility in selecting the cutoff frequency and sampling rate for data acquisition and has significant sampling rate errors $[2,24,25]$; the sensed data of the MTS310CA sensor board developed by Crossbow Technology, Inc., cannot well represent the physical response of the structure in the time domain and frequency domain [26].

Two methods are generally used for designing highperformance academic wireless sensors: the use of a commercially available platform interfaced with a customized sensor board and the use of a customized platform integrated with a customized sensor board. The first method uses commercial off-the-shelf motes, which is convenient and lends them to rapid development. However, the use of motes may restrict the performance and the operation of the platform. By contrast, the second method involves the development of a customized platform, which entails a complicated design and a long development cycle [13]. The main task of developing a wireless sensor is selecting appropriate components and connecting those components using the proper integrated circuit. At the same time, special control programs are configured to achieve presetting functions. In this section, academic wireless sensors explicitly proposed for use in bridge health monitoring are summarized.

In 1998, Straser and Kiremidjian [18] proposed a design of a low-cost wireless modular monitoring system (WiMMS) for civil structures by integrating a microcontroller with a wireless radio. This prototype represents the first major step by the structural engineering community towards decentralized data processing and wireless structural health monitoring [19]. The Motorola $68 \mathrm{HC11}$ microprocessor is chosen to control the remote wireless sensing unit. To achieve reliable wireless communication, a Proxim Proxlink MSU2 wireless modem operating on the 902-928 MHz ISM band is used. In 2001, the WiMMS was improved by Lynch et al. [15, 27, 28] with emphasizing the power of the computational core. The 8bit Atmel AVR AT90S8515 enhanced RISC (reduced instruction set computer) microcontroller is selected. Capable of eight million instructions per second, the microcontroller has high computational throughput without consuming large amounts of power. The Texas Instruments ADS7821 16-bit $\mathrm{ADC}$ with a maximum sample rate of $100 \mathrm{kHz}$ and $80 \mathrm{~mW}$ power consumption is used to translate analog signals to a digital format for processing. In 2003, this wireless sensor was further improved with the goal of low power consumption but high computational performance [29-31]. A dual-processor computational core design was proposed. The low-power 8-bit Atmel AVR AT90S8515 microcontroller is utilized for overall unit operation and real-time data acquisition, and the 32-bit Motorola MPC555 PowerPC is integrated to execute intensive data processing algorithms at a clock rate of $20 \mathrm{MHz}$. When the two microcontrollers are turned on, the AT90S8515 consumes $40 \mathrm{~mW}$ of power and the MPC555 (at $20 \mathrm{MHz}$ ) consumes $330 \mathrm{~mW}$. In sleep mode, the two microcontrollers consume $12 \mathrm{~mW}$, respectively. The modified wireless sensor is shown in Figure 3(a) [19]. And in 2005, the ADC was replaced with Texas Instrument ADS8341 16-bit ADC by Wang et al. [32-34]. An additional $128 \mathrm{kB}$ of static random access memory (SRAM) is interfaced with the microcontroller for the storage of measurement data. The most attractive feature of the wireless sensing unit design is its wireless radio. The MaxStream 9XCite wireless modem with $900 \mathrm{MHz}$ radio band and maximum transmission speed $38.4 \mathrm{kbps}$ is used. The communication range of the radio is $300 \mathrm{~m}$ line-of-sight, yet the radio only consumes $250 \mathrm{~mW}$ when transmitting, $150 \mathrm{~mW}$ when receiving, and less than $5 \mathrm{~mW}$ when idle. With efforts to further reduce the size of the wireless sensor, the electrical circuit is redesigned and printed on a compact two-layer circuit board. When fully assembled, the wireless sensor is $10 \times 6.5 \times 4 \mathrm{~cm}^{3}$ and is powered by five AA batteries, as shown in Figures 3(b) and 3(c). But the efforts have not stopped. First, the wireless communication system is renovated by the Chipcon CC2420, which is a single-chip IEEE 802.15.4 compliant radio capable of providing communication with ranges adequate for civil infrastructures [35]. The second significant improvement is the use of a four-layer circuit board, replacing the more limited twolayer boards. The four-layer boards allow for more compact designs, have the ability to devote the internal layers of the board to power and grounding planes, and provide a higher resolution for those channels. The latest version of the wireless sensor is named Narada, as plotted in Figure 3(d). Then, the Narada is used for executing many decentralized data processing algorithms proposed by Zimmerman et al. $[36,37]$.

Heo et al. [38] have designed a wireless MEMS-based accelerometer system, called SWMAS (Figure 4). This system consists of a sensor system module, a control and processing module, and a wireless modem module. The ADXL210 manufactured by Analog Devices is chosen for acceleration measurement because it could be used with the microcontroller directly without A/D converters. A low-power 8-bit microcontroller, Atmel AVR ATmega128 RISC, is selected for control of the data acquisition operation of the SWMAS. An external memory of 32 kbyte capacity is attached on the module to overcome the limit of SRAM. The CMRS232 is integrated as the communication module. Based on calibration test results, performances of SWMAS were shown to agree fairly with values of the datasheet. In a freevibration experiment using a cantilever beam, the response of SWMAS is in good agreement with the reference accelerometer. Subsequently, the SWMAS was improved to make it suitable to measure the response from structures such as very flexible bridges which have low amplitude and frequency of vibration signals [39]. First, the ADXL210 was replaced by the ADXL203. Secondly, the microcontroller was set at the noise reduction mode to get rid of the internal electric noise of the microcontroller occurring during A/D conversion. A sample test showed that the improved SWMAS could eliminate $70 \%$ of the noise. 


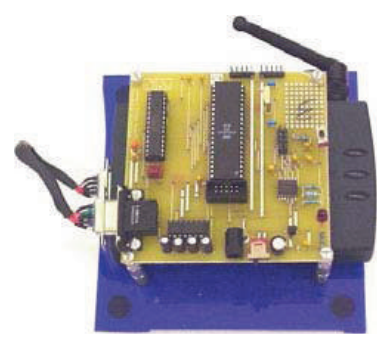

(a) Wireless sensor proposed by Lynch et al. in 2003 [29]

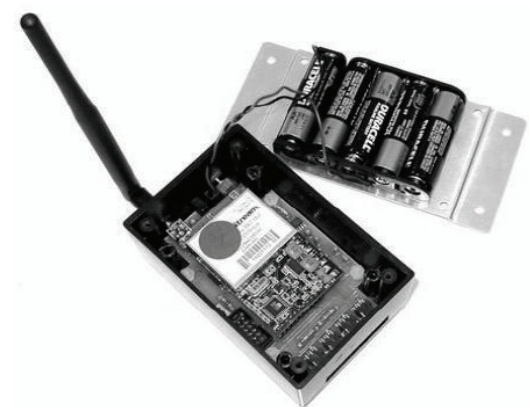

(c) The enclosed two-layer circuit board with batteries [34]

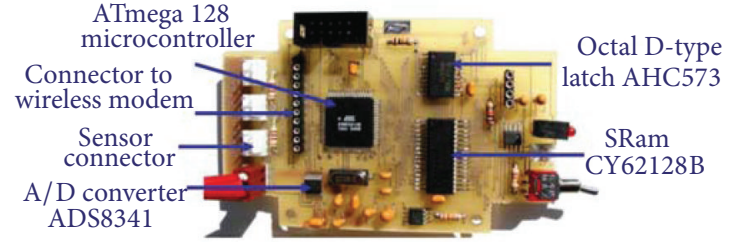

(b) The two-layer circuit board [34]

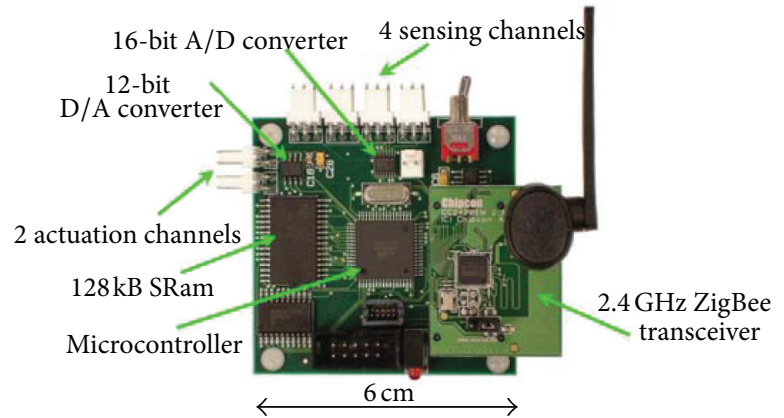

(d) Narada wireless sensing prototype [37]

FIGURE 3: Prototypes of wireless sensor.

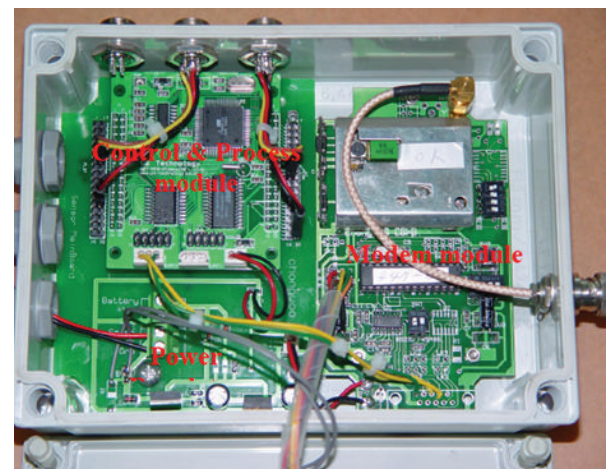

FIgURE 4: Prototype of SWMAS [38].

Park et al. [40] have described a wireless sensor, DuraNode, for structural monitoring. DuraNode has two low-power microcontrollers. One of the microcontrollers is dedicated to sampling the output of the accelerometers at a constant rate. The other microcontroller controls an 802.11b WLAN card and handles communication-related tasks such as forwarding incoming data packets to the next hop as well as sending out local data samples. These two microcontrollers communicate with each other using Serial Peripheral Interface (SPI). Each one of the two microcontrollers is the PIC18F8720. It has 64 kbytes of flash program memory, 3328 bytes of on-chip SRAM, an EEPROM of 1 kbytes, and 8 channels of 10-bit ADCs. The sensor circuitry consists of MEMS-type sensors and signal conditioning circuitry. Three MEMS-type SD1221 accelerometers are installed for sensing vibrations in the $x$-, $y$-, and $z$-axes. The bandwidth and coverage of the 802.11b WLAN is up to $11 \mathrm{Mbps}$ and 100 meters (outdoor, $1 \mathrm{Mbps})$, respectively.

Based on the commercial wireless sensor platform, Tmote Sky, Joshi et al. [41] have developed a sensor board with strain gauges. Tmote Sky developed by researchers at the University of California at Berkeley and marketed by MoteIV Corporation is a platform for extremely low power, high data-rate, sensor network applications designed with the dual goal of fault tolerance and development ease. Tmote Sky has on-chip RAM 10 kB, an IEEE 802.15.4 radio, and an integrated onboard antenna providing up to a 125-meter range. Tmote Sky offers a number of integrated peripherals including a 12-bit ADC and DAC, Timer, I2C, SPI, and UART bus. The microcontroller used in Tmote Sky is MSP430f1611, which has a 16-bit RISC processor, memory mapped analog peripherals, and memory mapped digital peripherals. The Chipcon CC2420 radio is included on the platform. The strain gauge used for this wireless sensor board has a nominal resistance of $1000 \Omega$ with a gauge factor of 2.105. A Wheatstone bridge is employed to convert the small resistance change in a strain gauge to voltage fluctuation. The PGA309, a programmable amplifier from Texas Instruments, is used in the signal conditioning circuit. PGA309 has basically two amplifiers: a front-end amplifier with gain selectable from 4 to 128 and an output amplifier with selectable gain of 2 to 9 . It is also interfaced with an external EEPROM where some of the configuration data is stored and where the temperature compensation lookup table is also stored. Figure 5 displays the strain sensor board connected to a Tmote Sky. 


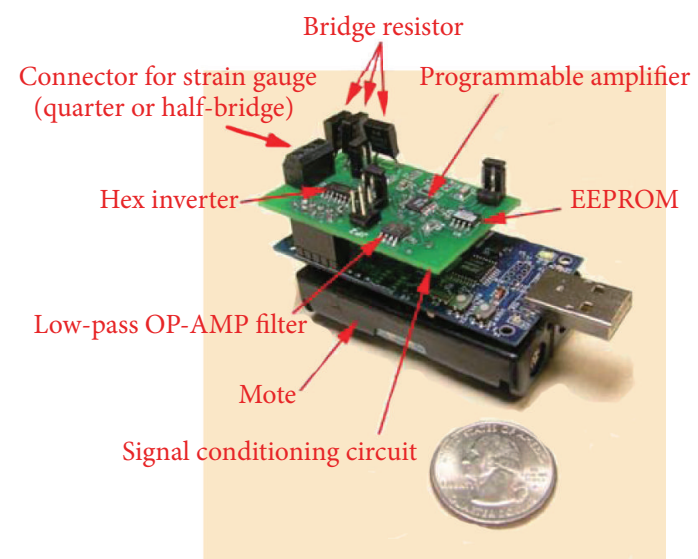

FIgURE 5: The strain sensor board connected to a Tmote Sky [41].

Using the MicaZ mote as the wireless sensor platform for control and communications, Kim et al. [12, 42] have designed an accelerometer sensor board for the application of bridge health monitoring. Figure 6(a) is a schematic of the major components of the board, and Figure 6(b) is the prototype of the accelerometer sensor board. The board has four independent accelerometer channels monitoring two directions (vertical and transverse) and a thermometer to measure temperature for compensation purposes. For the measurement of low-level and high-level accelerations, two commercially available MEMS accelerometer sensors are used each in two directions. Low-amplitude ambient vibrations, due to wind loading and traffic, are resolved by a two-dimensional Silicon Designs 1221L accelerometer. A low-cost ADXL202E two-dimensional accelerometer was used to monitor stronger shaking as might be expected from earthquake excitation. Tests show that the Silicon Designs accelerometers have a hardware noise ceiling of $10 \mu \mathrm{g}$, which is small enough to resolve signals with amplitude of a few hundreds of $\mu \mathrm{g}$. Each channel from the MEMS accelerometers provides an analog voltage that is fed to a single-pole antialiasing low-pass filter with a cutoff frequency of $25 \mathrm{~Hz}$ for the reason that even very high vibration models of a long-span bridge application have frequencies well below the antialiasing filter. The filtered analog signal is fed to a 16bit ADC for each of the four channels. A voltage regulator is contained on the sensor board to provide a constant $3 \mathrm{~V}$ output for the mote and a constant $5 \mathrm{~V}$ output for the ratiometric accelerometers. The MicaZ has a $512 \mathrm{kB}$ flash memory, which can store up to 250,000 2-byte data samples and a $2.4 \mathrm{GHz}$ radio frequency Chipcon CC2420 transceiver with a hardware interface that can support commercially available bidirectional antennas. This sensor is then applied to the SHM of the Golden Gate Bridge.

Employing the original design of wireless sensor node by Wang et al. [32, 33], Park et al. [43] have designed an acceleration-based sensor board (Acc-SSN) by modifying an antialiasing filter, MEMS accelerometer, and wireless radio capacity and an impedance-based wireless sensor board (Imp-SSN) based on the primary work of Mascarenas et al. [11], as shown in Figure 7. The Acc-SSN consists of eight components: power supply, MEMS accelerometer, coupling capacitor, amplifier, antialiasing filter, ADC, microcontroller, and wireless radio. The MEMS accelerator SD1221 is selected in the Acc-SSN. The coupling capacitor was designed by using a high-pass filter with a cutoff frequency of $0.1 \mathrm{~Hz}$. An operational amplifier is used to amplify low-level signals such as ambient vibration signals of civil structures. An 8thorder Butterworth low-pass filter with a cutoff frequency of $100 \mathrm{~Hz}$ is designed for SHM applications in civil structures. The four-channel 16-bit ADC ADS8341 (Texas Instruments, Inc.) is utilized as $\mathrm{ADC}$, and the 8-bit microcontroller ATmegal28 with low power consumption and low cost is selected as a microcontroller. The $2.4 \mathrm{GHz}$ frequency XBee (Digi International, Inc.) is chosen as the wireless radio, of which the outdoor line-of-sight range is up to $100 \mathrm{~m}$. The impedance chip of the Imp-SSN is the AD5933, which has the following embedded multifunctional circuits: function generator, digital-to-analog (D/A) converter, currentto-voltage amplifier, antialiasing filter, ADC, and discrete Fourier transform (DFT) analyzer. The AD5933 outputs real and imaginary values of impedance for a target frequency of interest and transfers the values into a microcontroller.

Adopting the commercial wireless sensor platform, Imote2, researchers at the University of Illinois at UrbanaChampaign (UIUC) [2] have developed a series of sensor boards or interface boards to meet the requirements of bridge health monitoring. The Imote2, developed by Intel, is wellsuited to the demands of SHM applications. It has a low-power X-scale processor (PXA27x), whose speed can be selected based on application demands and power management, ranging from $13 \mathrm{MHz}$ to $416 \mathrm{MHz}$. It incorporates a Chipcon 2420802.15 .4 radio with an onboard antenna. The onboard memory of the Imote 2 is the feature that sets it apart from other wireless sensor platforms and enables the intense onboard calculation required for SHM applications, as well as storage of longer measurements. It has $256 \mathrm{kB}$ of integrated RAM, $32 \mathrm{MB}$ of external SDRAM, and $32 \mathrm{MB}$ of flash memory. The Imote2 does not possess intrinsic sensing capabilities but rather provides a flexible platform for a range of sensing applications. The Structural Health Monitoring Accelerometer (SHM-A) board which uses a 3axis analog accelerometer (LIS344ALH), a temperature and humidity sensor (Sensirion STH11), the light sensor (TAOS 2561), a gain difference amplifier (AD628), and a Quickfilter (QF4A512) has been designed, as shown in Figure 8 [44]. The board interfaces with the Imote2 via SPI I/O. The LIS344ALH, which has better performance than other comparably priced sensors [45], is a capacitive-type MEMS accelerometer with DC to $1500 \mathrm{~Hz}$ measurement range and offers an excellent price/performance ratio. The measurement range, resolution, and noise density of this accelerometer are $\pm 2 \mathrm{~g}, 0.66 \mathrm{~V} / \mathrm{g}$, and $50 \mu \mathrm{g} / \sqrt{\mathrm{Hz}}$, respectively. The QF4A512 employs a versatile 4-channel, 16-bit resolution ADC and a programmable signal conditional with a user-selectable sampling rate and programmable digital filters. A software driver for the SHM-A board was developed in TinyOS to control the functions of the QF4A512 such as loading the filter coefficients, allocating memory, timestamping, writing data, and onboard temperature compensation. The SHM-A 


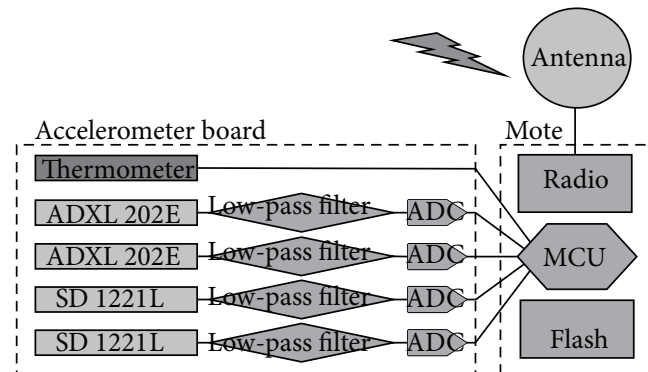

(a) Accelerometer sensor board block diagram

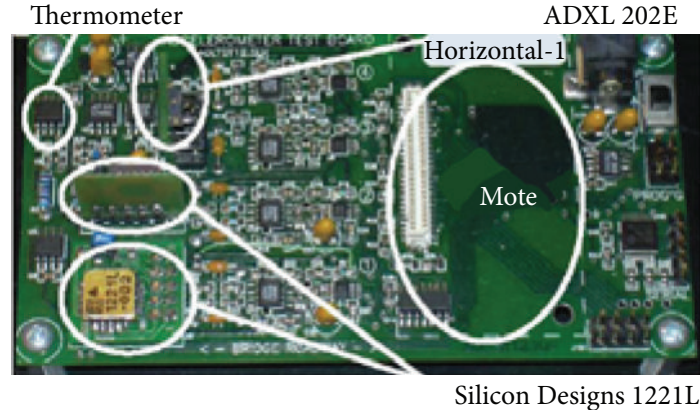

(b) Prototype of accelerometer sensor board

FIgURE 6: Accelerometer sensor board [12].

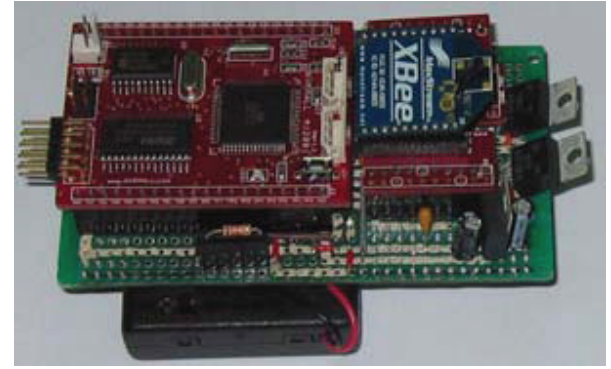

(a) Prototype of developed Acc-SSN

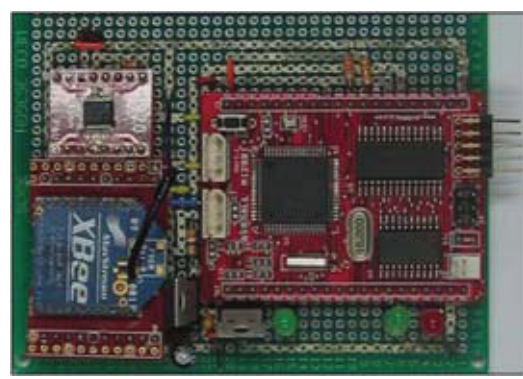

(b) Prototype of developed Imp-SSN

FIgURE 7: Wireless sensor proposed by Park et al. [43].

board provides excellent resolution for bridge health monitoring and is already commercialized by MEMSIC as the ISM400. Jo et al. $[2,46]$ have improved the resolution of the SHM-A board and developed a low-noise high-sensitivity accelerometer board (SHM-H) for the Imote2 platform to measure low-level ambient vibrations of structures, whose levels are typically on the order of $1 \mathrm{mg}$ or less. Figure 9 shows the details of the SHM-H board. The $z$-axis of the SHMA board is replaced with a low-noise and high-sensitivity sensor, the Silicon Designs SD1221L-002. To support the lownoise performance of the accelerometer, the resolution of the QF4A512 ADC was improved by reducing the span of the ADC from $\pm 2 \mathrm{~g}$ to $\pm 1.2 \mathrm{~g}$. The RMS noise of the SHM$\mathrm{H}$ sensor board is $0.05 \mathrm{mg}$ over $15 \mathrm{~Hz}$ bandwidth $(0.08 \mathrm{mg}$ over $70 \mathrm{~Hz}$ bandwidth), which is about $15 \%$ of the SHM-A board's noise level. Then, Spencer Jr. et al. [2, 47] designed a general purpose data acquisition board (SHM-DAQ board) for the Imote2 platform to interface with external analog sensors and digital sensors. Any type of analog sensor having DC voltage signals, whose ranges are $0 \sim 5 \mathrm{~V}$ or $-5 \sim 5 \mathrm{~V}$, and I2C digital sensors can be accommodated with Imote2 through the terminal block of this board. In 2010, Spencer Jr. et al. developed a strain sensor board (SHM-S board) for structural strain monitoring, as can be seen in Figure 10. This sensor board includes a signal amplification circuit and a Wheatstone bridge circuit to enhance the readability of the low level of strain gage signals. This sensor board is designed for stacked use with the SHM-DAQ board or SHM-A board, which is also plotted in Figure 10. The resolution of SHM-S board is about $1 \mu \varepsilon$ strain at $100 \mathrm{~Hz}$. Besides, Spencer Jr. et al. have designed a pressure sensor board (SHM-P board) to provide wind pressure measurements using the AMSYS 5812 high-precision analog pressure sensor and a digital-to-analog converter (DAC) (SHM-D2A board) to support the output of signals using the TI-DAC8565.

The Laboratory for Intelligent Infrastructure and Transportation Technologies (LIITT) at Clarkson University has proposed a wireless sensor system (WSS) for concurrent measurement of both static and dynamic structural responses through strain transducers, accelerometers, and temperature sensors, as shown in Figure 11 [48]. The developed wireless sensor node incorporates the Tmote Sky wireless sensor network platform. The onboard Chipcon CC2420 $2.4 \mathrm{GHz}$ transceiver offers an effective data rate of $250 \mathrm{kbps}$ and low power consumption. An integrated 12-bit successive approximation register $\mathrm{ADC}$ provides eight external channels and a greater than $200 \mathrm{ksps}$ maximum conversion rate. To facilitate high-resolution acquisition of distributed acceleration measurements for modal analysis of structures, a custom signal conditioning subcircuit provides analog low-pass filtering, digital offset correction, and digitally programmable gain for up to two single-ended analog signals. An independent signal conditioning interface is provided for the acquisition of differential sensor signals, such as Wheatstone bridge resistive sensors like strain transducers, load cells, pressure sensors, and displacement sensors. 


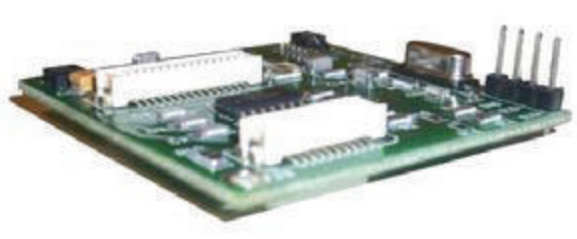

(a) Perspective

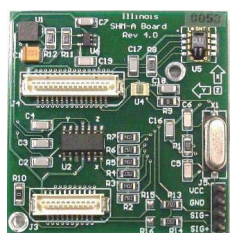

(b) Top view

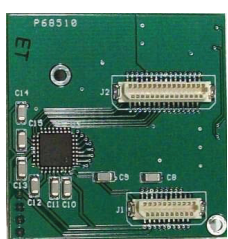

(c) Bottom view

Figure 8: SHM-A board [2].

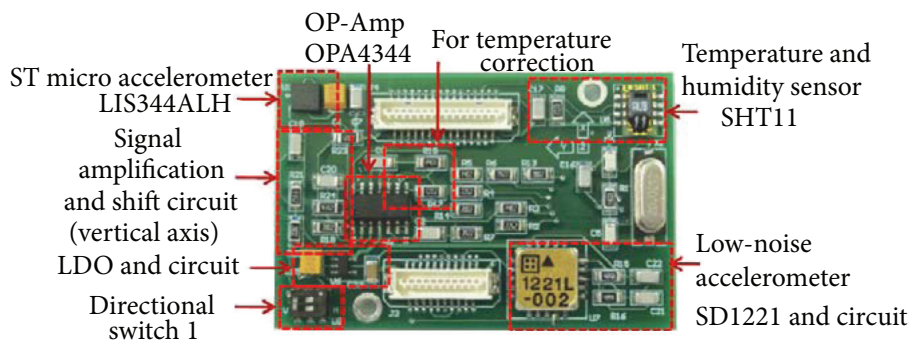

(a)

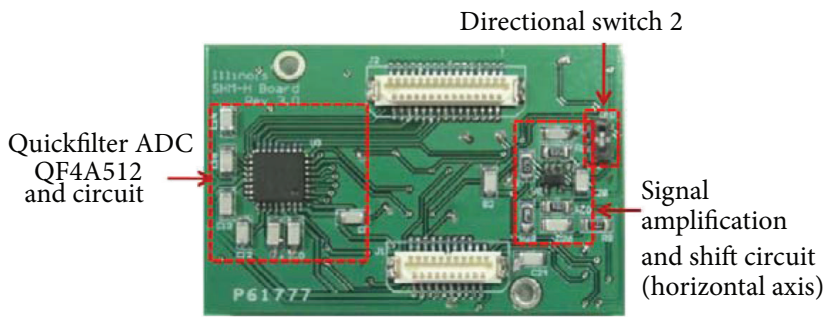

(b)

Figure 9: Top view (a) and bottom view (b) of SHM-H sensor board (revision 3) [46].

In 2010, Chen and Liu [49] developed a wireless sensor node. The sensor node consists of three circuit boards: the sensing board, the Gumstix board, and a wireless communication board. The Gumstix board communicates with the sensing board through I2C bus and connects to the wireless communication board through a parallel port. The Gumstix embedded computer is one of the world's smallest full function miniature computers with a size of $20 \mathrm{~mm} \times$ $80 \mathrm{~mm} \times 8 \mathrm{~mm}$. The product is based on the Intel PXA-255 processor with $\mathrm{X}$-scale technology and a Linux operating system. The Gumstix board has 64 MB RAM, 16 MB Flash, and $400 \mathrm{MHz}$ CPU speed and also provides external memory spaces. Two server programs, a remote secure shell server and a web server, are provided for the users to remotely access the computer. The sensor board consists of an ATmegal28L $\mathrm{CPU}$ for real-time data acquisition and communication with the Gumstix mother board, 16-bit ADCs and signal conditioning circuits for accelerometer and strain gage signal processing, an active sensing signal generator and response analyzer for active sensing with piezoelectric transducer (PZT) sensors/actuators, a ZigBee Module for low-power wireless communication, and an external SRAM for real-time data buffering.
Bocca et al. [20] have created a wireless sensor node, namely, ISMO-2 node, which originated from their previous ISMO node [3], as shown in Figure 12. The ISMO-2 node is based on the Sensinode U100 Micro.2420 sensor network platform. Its core is a TI MSP430F1611 MCU with $48 \mathrm{kB}$ of program and $256 \mathrm{~B}$ of data flash memory and $10 \mathrm{kB}$ of RAM. The platform also has an external $500 \mathrm{kB}$ serial data flash memory (M25P40 by STMicroelectronics). The microcontroller unit (MCU) provides one 12-bit ADC (up to 8 channels available) and two 12-bit DACs. The clock of the $\mathrm{MCU}$ and the bus runs at $8 \mathrm{MHz}$. The radio module is an IEEE 802.15.4 compatible Chipcon CC2420 transceiver. The node is equipped with a dedicated sensor board, in which a 3-axis digital accelerometer (LIS3LV02DQ by STMicroelectronics, $7 \times 7 \times 1.5 \mathrm{~mm}^{3}$ ) is soldered. The Micro. 2420 platform provides another 12-pin external connector to which a lowpower temperature and humidity sensor (SHT71 by Sensirion) is connected.

Chae et al. [9] have proposed a sensor module that includes an A/D board and a ZigBee board (Figure 13) that was termed as u-Node. The u-Node is powered by a battery and a solar cell to operate and is hard-cased for water resistance. The distinguished feature of this node is that the $16-b i t \mathrm{~A} / \mathrm{D}$ 


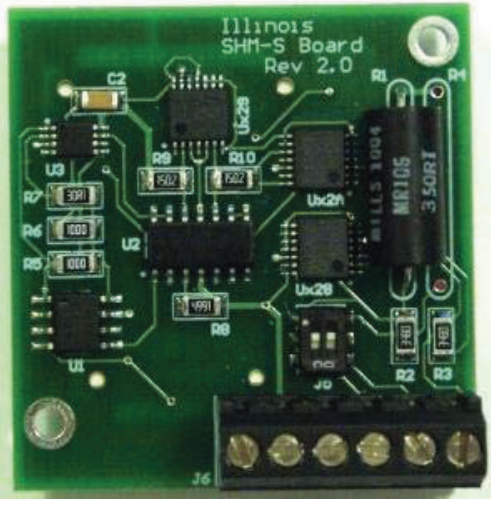

(a) Top view

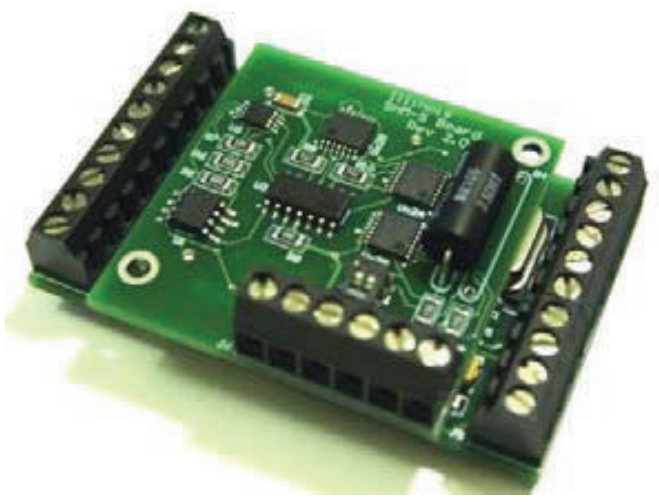

(b) With SHM-DAQ

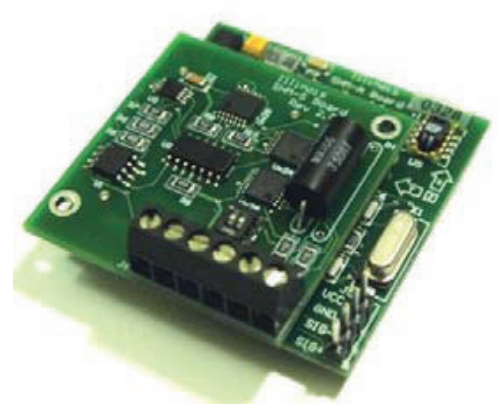

(c) With SHM-A

FIGURE 10: SHM-S board [2].

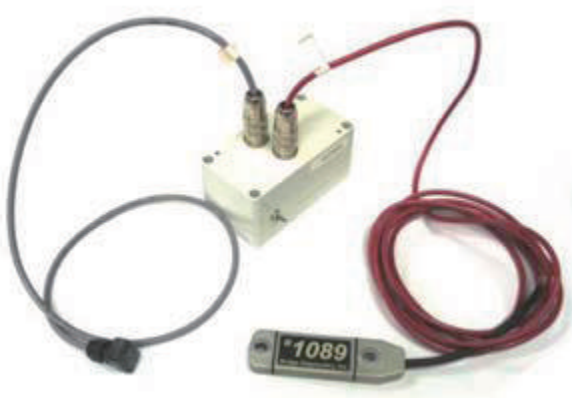

(a) WSS hardware with MEMS accelerometer

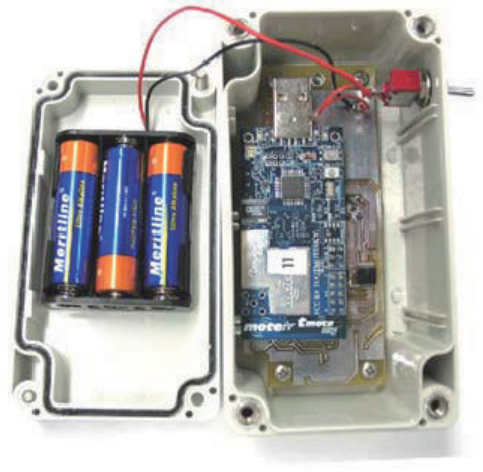

(b) WSS hardware with strain transducer

FIGURE 11: The WSS hardware [48].

board is compatible with the output of resistance, current, and voltage. Real-time clock (RTC) chips were used to ensure accurate monitoring times, with the Analog Devices AD7708 model for the ADC chip and an Atmel 128L for MCU. The A/D converting circuit is composed of a sensor interface, signal conditioning, A/D conversion, and power. Variety types of industrial sensors, such as accelerometer, strain gauge, thermometer, and wind gauge, can be used in this node. But the consistent and stable power supply to this node is a critical problem.

$\mathrm{Hu}$ et al. [13] have developed an S-Mote platform, an acceleration sensor board, and a strain sensor board to satisfy the requirements of bridge structural monitoring (Figure 14). The designed S-Mote improves the flexibility in connecting different sensor boards or in offering communication functions. The platform can collect different kinds of sensor signals, such as acceleration, temperature, and strain signals. S-Mote (Figure 14(a)), which is designed for SHM applications, is composed of four modules: microcontroller unit, RF module, power management unit, and sensor expansion pins. The ultralow-power microcontroller, MSP430F1611, was selected for S-Mote. The microcontroller runs on $1.8-3.6 \mathrm{~V}$. When running at $1 \mathrm{MHz}$ with a supply voltage of $2.2 \mathrm{~V}$, the microcontroller consumes $330 \mu \mathrm{A}$ current in active mode; off-mode operation reduces consumption to $0.2 \mu \mathrm{A}$. MSP430F1611 provides the largest on-chip (10 kB) RAM buffer, $48 \mathrm{kB}$ of flash memory, an integrated 12-bit $\mathrm{ADC}$, and a maximum conversion rate greater than $200 \mathrm{kbps}$. S-Mote has a 16-pin IDC expansion header for connecting sensor boards. Through exportation of I2C, UART, AD, and digital I/O over the expansion header, expanders can be used to attach different kinds of sensor boards. S-Mote uses the Chipcon CC2420 radio in a $2.4 \mathrm{GHz}$ band. The twodimensional Silicon Designs 1221L accelerometer is used in the acceleration sensor board, while multilevel amplifier technology is adopted to avoid the self-excited oscillation caused by excessive magnification. The foil strain gauge is chosen for the strain sensor board. An asymmetrical bridge circuit configuration is developed to compensate for temperature changes in the strain board.

Besides, other research groups have developed different wireless sensors to address the special demands of bridge health monitoring. Wang et al. [50] have designed a wireless sensor specifically for displacement and strain measurement using a polyvinylidene fluoride (PVDF) thin film sensor. Ruiz-Sandoval et al. [26] have developed an acceleration 


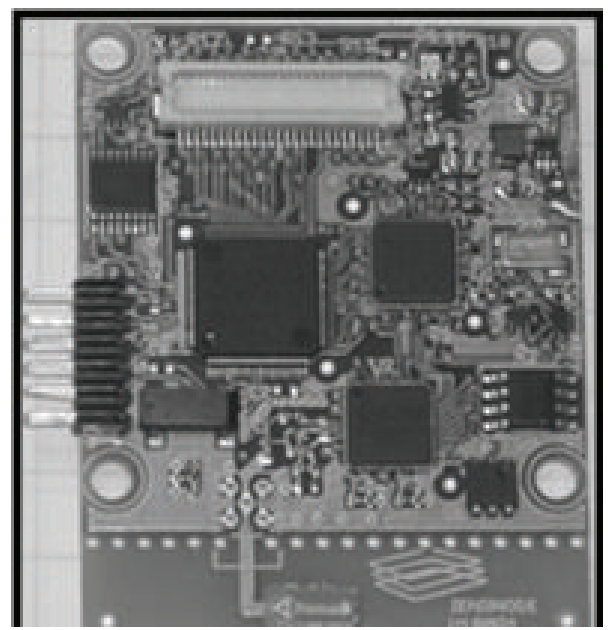

(a) Micro.2420

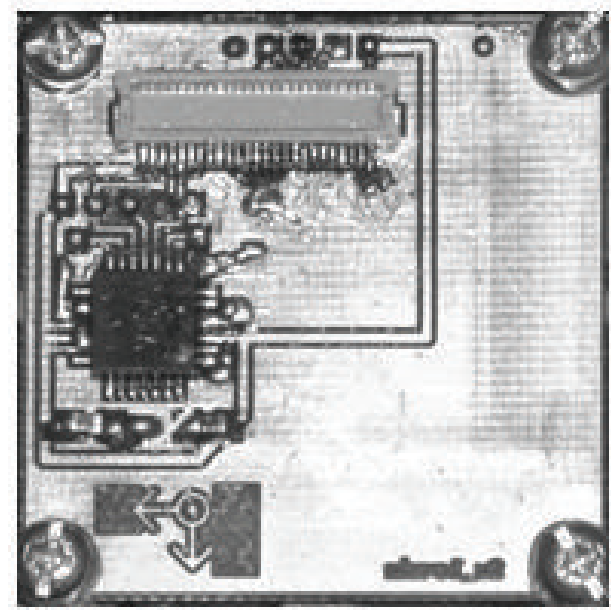

(b) Acceleration board

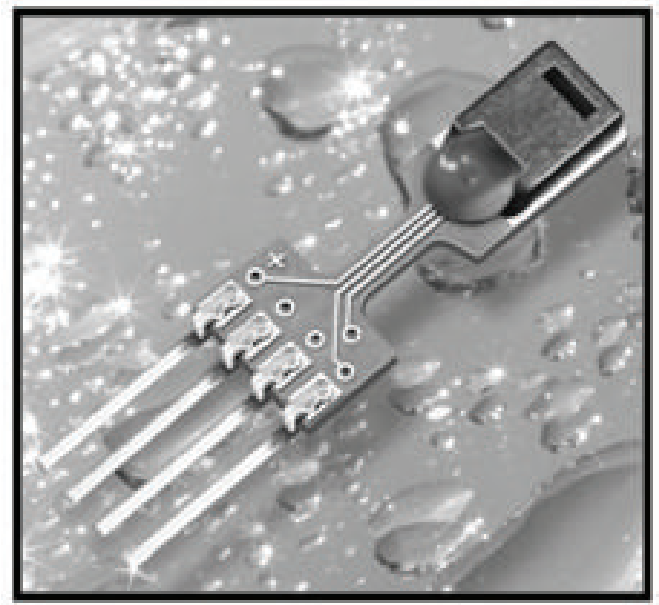

(c) Temperature/humidity sensor

FIgURE 12: The ISMO-2 node [20].

board and a strain board using commercial Mica motes for communication and control, but the system relies on singlehop wireless communication, which is not scalable to large structures. Loh et al. [51] have presented a multifunctional carbon nanotube-polyelectrolyte-based nanocomposite passive wireless sensor for SHM applications. Harms et al. [52] have developed a wireless sensor named SmartBrick, which is equipped with a Quad-Band GSM/GPRS modem. Yang et al. [53] have presented a wireless sensor node which emphasizes monitoring the long-term continuous static behavior of a structure. Taylor et al. [54] have designed a wireless impedance sensor node (WID3) for use in high-frequency impedance-based SHM and low-frequency vibration data acquisition. Min et al. [55] have developed in-field adjustable impedance-based wireless sensor nodes in which an integrated impedance converter (AD5933) is equipped.

It is worth to say that many proposed wireless sensors mentioned previously are platforms on which a variety of sensors can be interfaced. If the output of a sensor can meet the demands of the input of the wireless sensor platform, the sensor can be integrated with the wireless sensor platform. As a result, it is difficult to provide the exact structural parameters that can be measured by WSNs. Now, almost all structural parameters can be measured by WSNs, but the emphasis is placed on acceleration and strain.

3.2. Network Topology. The realization of operations in the WSNs-based bridge health monitoring system such as collecting data, transmitting data, and propagating orders relies completely on the WSNs. The network topology, which is used to organize wireless sensors in the network by routing and enable all sensors to cooperate with each other, is one of significant components in the WSN-based bridge health monitoring system. In general, according to the network topology, WSNs can be classified as a single-hop network and multihop network.

3.2.1. Single-Hop Network. Figure 15 shows the overview of the single-hop network, which is also called star topology. 


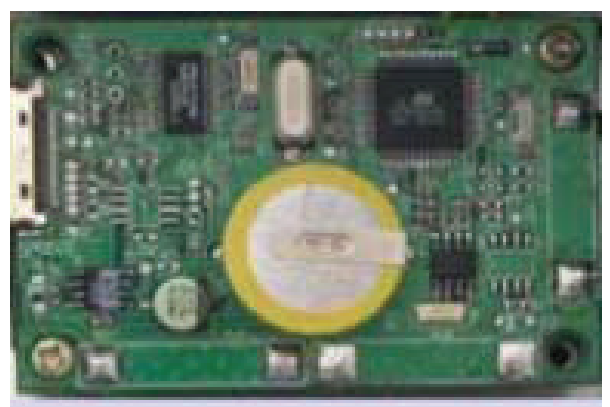

(a) A/D board

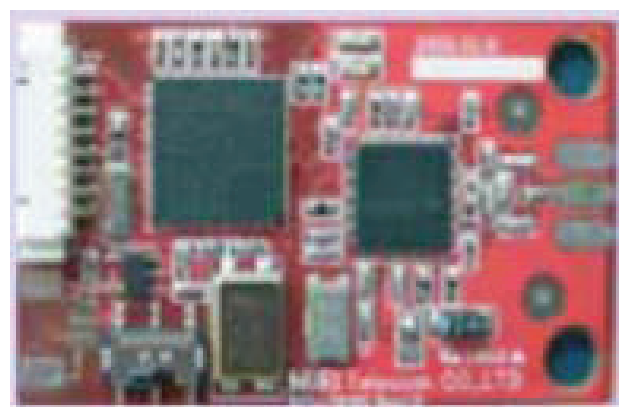

(b) ZigBee board

FIGURE 13: The u-Node [9].

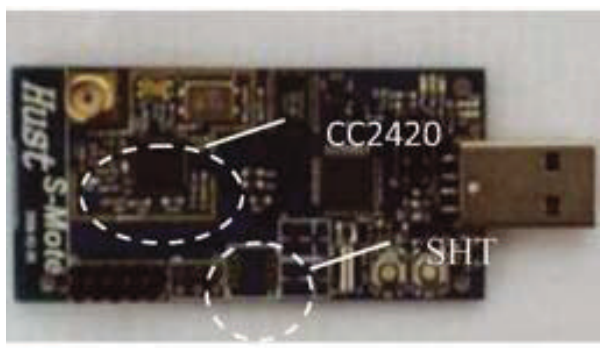

(a) S-Mote node

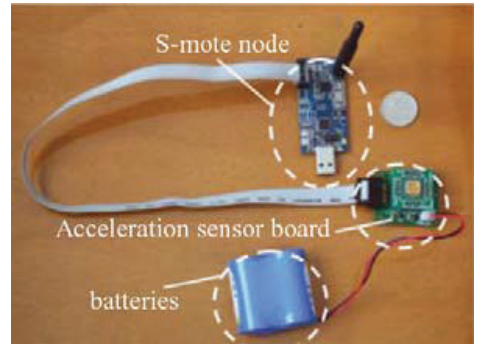

(b) S-Mote with acceleration board

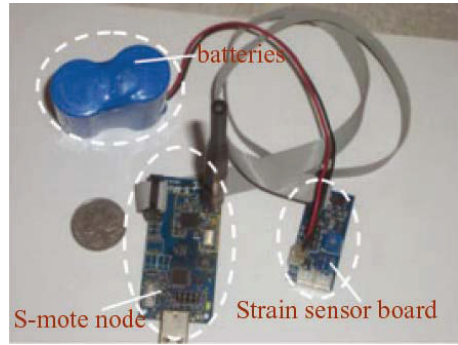

(c) S-Mote with strain board

FIGURE 14: The prototype of the wireless sensor proposed by $\mathrm{Hu}$ et al. [13].

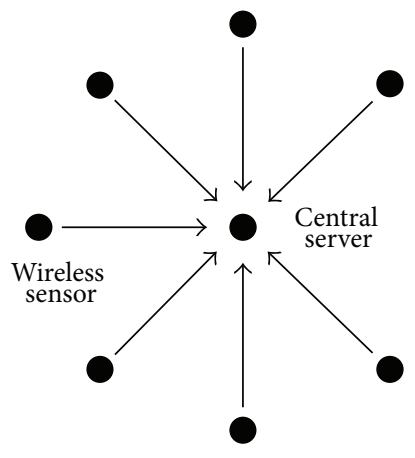

FIGURE 15: Single-hop network.

The central server is located in the center of the network. All wireless sensors are around the central server. Data are transmitted directly to the base station by a single hop. This network topology is very simple and robust. The failure of one node does not influence the operation of the whole network. But the data packets in a single-hop network are delivered one by one, the processing speed relied on the performance of the gateway and the central server. Furthermore, single-hop networks are spatially limited by the radio range and cannot span long distances without a large power supply. The singlehop network is fit for the monitoring of small-scale bridges like laboratory models $[3,9,56,57]$.

3.2.2. Multihop Network. The limited radio range of a general WSN using IEEE802.11 or IEEE802.15 wireless protocols, combined with the impact of various environmental effects on the radio transmission, makes direct communication between all nodes impractical especially for long-span bridges. The multihop network, which transfers data between nodes or from sensor nodes to the central server by multihop communication, becomes an attractive alternative. Multihop communication transmits data and commands between two end nodes that are not in the direct radio range, using intermediary nodes. Multihop routing is more complex because each node has to determine how to find the most efficient routing to forward packets to the central server and coordinate transmission of packets received from other nodes. The routing needs to be reconfigured dynamically for robustness if a node fails and is no longer able to serve as an intermediary. Large-scale deployment of WSNs on long-span bridges gives rise to the need for multihop communication to provide adequate wireless coverage. The multihop network can be further classified into four categories: mesh-type network, tree network, linear multihop network, and random multihop network, as shown in Figure 16.

In a mesh-type network, there is no predetermined central server. Any node can be temporarily appointed as the central server according to the needs of data transmission and data processing. The node can transfer data to any other nodes within the transmission range. This type of network enables realizing completely auto damage diagnosis without external interference if a proper embedded software is designed. But the routing is very complex and is difficult to carry out, so it is rarely applied in the WSNs-based bridge health monitoring system. 


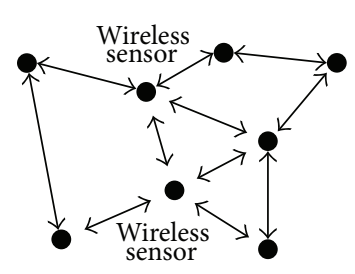

(a) Mesh-type network

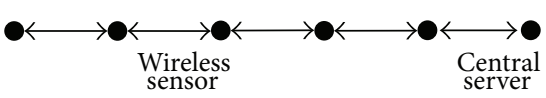

(c) Linear multihop network

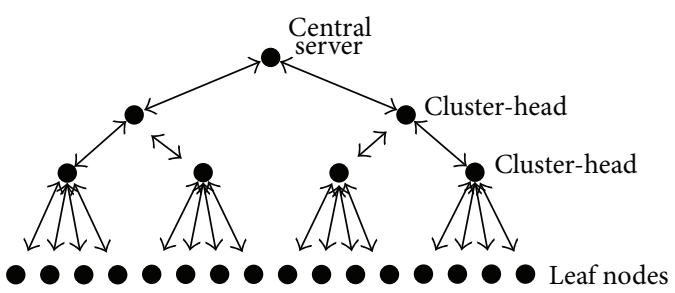

(b) Tree network

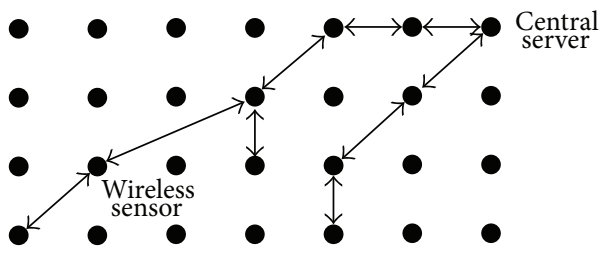

(d) Random multihop network

FIGURE 16: Multihop network.

The tree network is a type of multitiered network. The wireless sensors in the lowest level are named leaf nodes. The leaf nodes in WSN are assigned to different communities. In every community, there is a cluster-head, which is responsible for organizing communication and data processing within the community. And the communities can form a higherlevel community until the cluster-head is able to communicate with the central server directly. The data packs are delivered from leaf nodes to the cluster-head, and to the higher-level cluster-head, to the base station finally. The communication among communities can be permitted or forbidden on different occasions. More power source and high computational ability are required for the cluster-head for heavy data transmission load and busy data processing. Gao et al. [58] proposed a distributed computing strategy (DCS) for SHM based on this network topology.

The data are transmitted one by one in a linear multihop network. All wireless sensors in the network form only one data link. Intermediary nodes have three functions: data sensing, data receiving, and data delivery. The geometrical diagram of a linear multihop network is very similar to that of a bridge span, so it is fit for long-span bridge monitoring. But the successful data transmission depends on every intermediary node in the data link. The death of any intermediary node may invalid the data link. In addition, packet loss recovery may be problematic in a long data link. The implement of the wireless health monitoring system on the Golden Gate Bridge is based on this type of network [12]. The speed of data collection is reduced dramatically with the increasing of the data link length.

And in a random multihop network, the data packs of wireless sensors are transmitted randomly until they arrive to the base station. Every data link from the wireless sensor to the central server can be regarded as a linear multihop network. But the wireless sensors have many choices of the next station. The random multihop network provides the maximum flexibility of wireless sensors configuration, which can fit the monitoring of a structure with complicate geometry. Theoretically, this type of network can be scaled to an infinite area. But the nodes near the base station exhaust their power quickly for the large amount of transmitted data; "energy hole" is avoided uneasily [59].

3.3. Data Processing Technology. The bridge health monitoring assesses the safety and integrity of the structure based on the data measured by a variety of sensors. Since potentially problematic structural changes, such as corrosion, cracking, buckling, and fracture, all occur locally within a structure, responses from sensors close to the damaged site are expected to be more heavily influenced than those from sensors remote from the damage. As a result, to effectively detect arbitrary damage in a complicated structure, a dense array of sensors distributed over the entire structure will be required. Firstly, a tremendous amount of data is expected to be generated and managing this large amount of data is challenging. Secondly, data communication in WSNs is one of the most significant sources of power consumption and network failure, and reducing wireless communication becomes an important factor in prolonging the lifetime of WSNs. Thirdly, transferring a large amount of data will result in severe data congestion in the WSNs due to the limitation of wireless communication bandwidth. Because of the above three reasons, data processing technology, which assists in dealing with the large amount of data that is generated by a monitoring system, becomes an important issue in successfully implementing a large-scale WSN for evaluating the performance of bridges. Such an approach provides an adaptable sensor with selfdiagnosis and self-calibration capabilities, thus reducing the amount of information that needs to be transmitted over the network.

In recent years, many data processing programs have been developed in the open-source environment of TinyOS, which is one of the most widely utilized operation systems (OSs) for deeply embedded wireless sensor networks. TinyOS is written in the high-level programming language nesC, which is based 


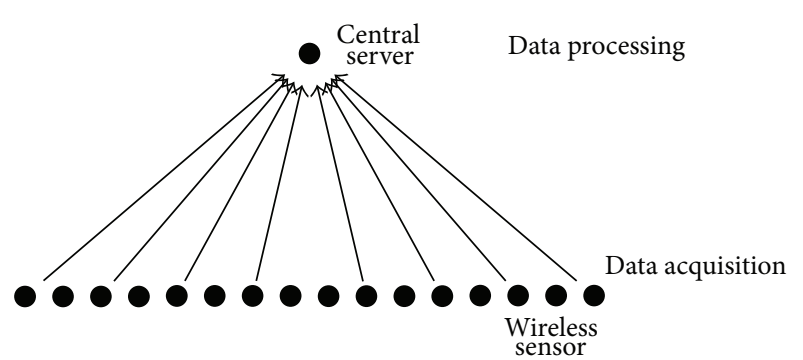

(a) Centralized processing

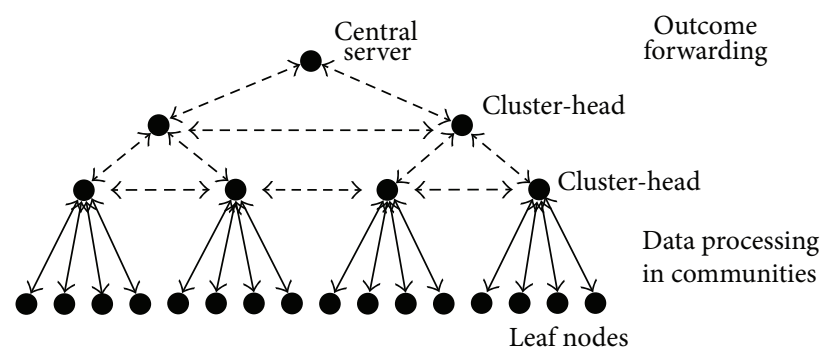

(c) Coordinated processing [2]

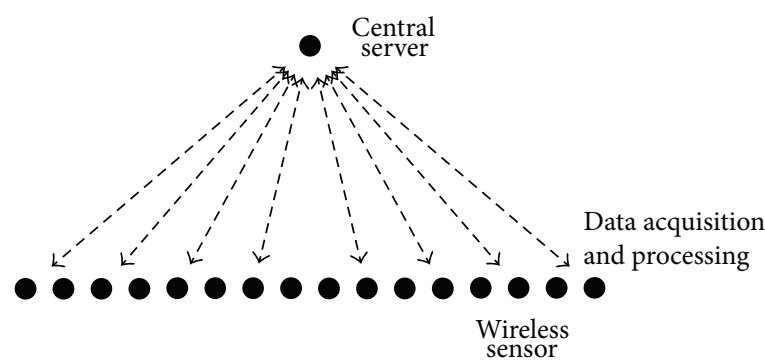

(b) Independent processing

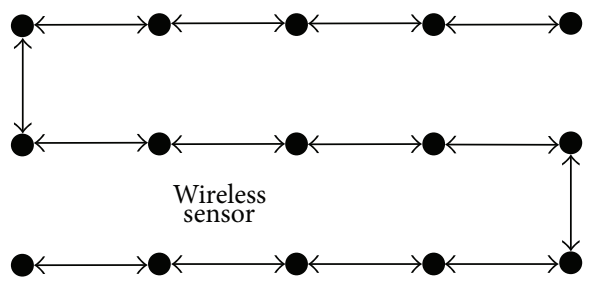

(d) Parallel processing

FIGURE 17: Data processing strategy.

upon the $\mathrm{C}$ programming language. Unfortunately, it is a very challenging environment for nonprogrammers to develop network control and application software. Extensive expertise is required to develop SHM applications in TinyOS $[2,19]$. In general, data processing technology can be classified into four categories: centralized processing, independent processing, decentralized processing, and parallel processing. Figure 17 presents the data flow of different data processing algorithms. The latter three belong to the distributed processing strategy. In the distributed computing strategy, the sensing data are firstly processed locally by using the onboard microprocessor of the wireless sensor, and then unnecessary information is eliminated through data aggregation to efficiently utilize the network, reducing the information that needs to be transferred back to the central server. Indeed, the distributed processing strategy, which can utilize distributed computational capability efficiency, is regarded as another key motivation besides low cost that promotes the SHM from a traditional wired network to a wireless network and represents a new data management paradigm associated with SHM.

\subsubsection{Centralized Processing. Centralized processing} (Figure 17(a)), like the traditional wired bridge health monitoring system, requires that all data be sent back to a central server for further processing. Owing to the high-performance computer of the central server, all existing structural condition evaluation algorithms can be employed in this data processing strategy. However, in this strategy, the amount of wireless communication required in the network becomes costly in terms of excessive communication times and the associated power it consumes as the network size increases. For example, a wireless sensor network implemented on the Golden Gate Bridge that generated $20 \mathrm{MB}$ of data (1600 seconds of data, sampling at $50 \mathrm{~Hz}$ on 64 sensor nodes) took over nine hours to complete the communication of the data back to a central server $[42,60]$.

Because of power requirements and bandwidth limitations, transferring all the measured information to a central server using WSN is difficult. Furthermore, the high data load and low efficiency of centralized processing cannot meet the demand of timely identifying structural damage. So processing data locally or dispersedly through the sensor's embedded microprocessor has received considerable attention. This strategy is also a radical departure from the conventional approach to monitoring structures.

3.3.2. Independent Processing. Independent processing, as shown in Figure 17(b), utilizes the computational power of every local sensor node to process collected data and requires no communication between sensors. The embedded algorithms on local wireless sensors collect and process raw measurement data available at each sensor with the processed results transmitted one by one to a central server. The central server further extracts useful parameters from different processed results and can also send them back to the wireless sensors when necessary. Using the data exchange between wireless sensors and the central server, the complicated damage diagnosis algorithms would be realized. Because of no data fusing, the implement of independent processing is relatively simple. The biggest challenge is how to modify the algorithms so that they can be realized in a computational core with limited power and executed in the special OS.

Early in 2002, the Cooley-Tukey implementation of the FFT was successfully embedded in the computational core of a wireless sensing unit by Lynch et al. [29, 61, 62]. The embedded FFT is utilized during field deployment of 
the wireless monitoring system to provide the frequency response functions (FRFs) of instrumented structures. The wireless sensor is shown to provide identical results compared to those generated by MATLAB using the same time-history data. Then, the FFT has been coded to be executed by both the Motorola MPC555 and Atmel AVR microcontrollers and used to accurately identify the primary model frequencies of various laboratory and field structures. In addition, the peakpicking (PP) scheme that can identify modal frequencies from peaks in the Fourier spectra was also embedded in the wireless sensor. The following year, they executed the autoregressive (AR) model and autoregressive model with exogenous inputs (ARX) prediction (AR-ARX) model in the wireless sensing unit computational core to automatically detect damage of a structure [62-64]. The AR-ARX model takes the residual error of the fitted AR model to compare it with the database of AR-ARX model pairs previously calculated as a damage sensitive feature. In this strategy, only the AR or ARX model coefficients were wirelessly transmitted, and, therefore, the energy consumed for transmission was considerably reduced. The laboratory example shows that the computational core of the wireless sensing unit has successfully executed the AR-ARX model for detecting the possible existence and location of damage in the system. The algorithm of AR coefficients estimation was also embedded in another wireless sensor unit by Kiremidjian et al. [65].

Caffrey et al. [66] have proposed an algorithm to detect damage of a structure by assessing change in modal frequencies and signal energy contained in each corresponding mode. Fourier spectra of structural acceleration time histories are calculated in each wireless sensor unit. After the modal frequencies and modal signal energy contributions are determined in the wireless sensor units, they are transmitted to the host computer to perform damage assessment. The proposed approach is verified by detecting the presence of damage of the IASC-ASCE SHM Benchmark Structure. Hashimoto et al. [67] have embedded a wavelet-based damage detection method in a wireless sensor unit. The continuous wavelet transform is used as an algorithm of data processing. But their strategy is only fit for a single-degreeof-freedom (SDOF) system. Additionally, Chintalapudi et al. [68] embedded a distributed damage detection algorithm in wireless sensors by identifying frequency shifts caused by damage.

Cho et al. $[69,70]$ have explored a vibration-based tension force estimation method proposed by Zui et al. [71] using a PP algorithm and embedded it in the computational core of a wireless sensor. Welch's method to average Fourier spectra from the segments of a whole time-history signal is employed to remove the nonstationarity of a short-duration acceleration record, which is a limit of the memory-constrained wireless sensor. A series of laboratory tests are conducted on a slender braided steel cable with a variety of cable sags and tension forces. Excellent agreements have been found between the actual tensions and those estimated by the present wireless system. However, the vibration-based tension force estimation method derived by Zui et al. utilizes three lower natural frequencies of a cable. The method may be impractical if vibration of the resisting structure is exciting the cable, which is called cable-deck interaction. The cabledeck interaction is dominant in the lower natural frequency region, which may distort the vibration signal of a cable, disabling reliable automated peak-picking.

Castaneda et al. [72] and Hackmann et al. [73] have tried to detect damage of a structure by the damage location assurance criterion (DLAC) method. The DLAC method is based on a correlation analysis between identified natural frequencies from measured data and analytical ones in each simulated damage pattern. Acceleration time-history records are transformed to frequency domain by applying the FFT. PSD functions are then calculated. Finally, a curve fitting technique is applied to the PSD function to determine resonant frequencies locations. A set of intermediate curve fitting parameters calculated on a local wireless sensor unit are then transmitted wirelessly to the base station where final roots extraction is finished and DLAC values are reported. It is demonstrated that the decentralized DLAC method can identify damage effectively. But the DLAC method has some limitations because it is not applicable to multiple damage scenarios or symmetrical structures.

Spencer Jr. et al. [60, 70, 74] have provided a library of numerical functions that are common to many SHM algorithms including FFT, singular value decomposition (SVD), Eigenvalue analysis, PSD estimation, and PP. All of those algorithms were modified to be used on the Imote 2 and formed different open-source services. Researches all over the world can download them for free, which releases a lot of elementary work for performing high-level decentralized data processing strategies and damage diagnoses. For example, Sim et al. [75] have embedded the cable tension estimation that autonomously interrogates cable tensions in the Imote2 platform based on the PSD algorithm and the PP algorithm. This method of cable tension estimation uses the closed form relationship between natural frequencies and the tension force proposed by Shimada [76], which only requires the natural frequencies of a high model of the cable to avoid the cable-deck interaction.

Most recently, an agent-based computing paradigm has emerged in which all wireless sensor units in a homogenous WSN are utilized in a completely parallel and decentralized manner in order to solve complex engineering problems autonomously and without the need for any higher-level coordination. This type of in-network computation has been applied to various SHM problems including modal estimation, model updating, and task scheduling. Chen and Liu [49] presented a mobile agent middleware that allows a sensor network moving computational programs to the data source. The mobile agent code is a regular $\mathrm{C}$ program. The AR-ARX is used for damage diagnosis. The validation example shows that the presented mobile agent approach can successfully deploy the AR-ARX damage diagnosis algorithm on distributed sensor nodes.

Hsu et al. [77] embedded a frequency response function change method (FRFCM) in the computational core of the wireless sensing units to realize online damage localization and quantification of a structure. After the acceleration time history $y_{i}(t)$ is measured in the $i$ th wireless sensor unit (WSU), the Fourier spectrum $Y_{i}(\omega)$ is calculated by 


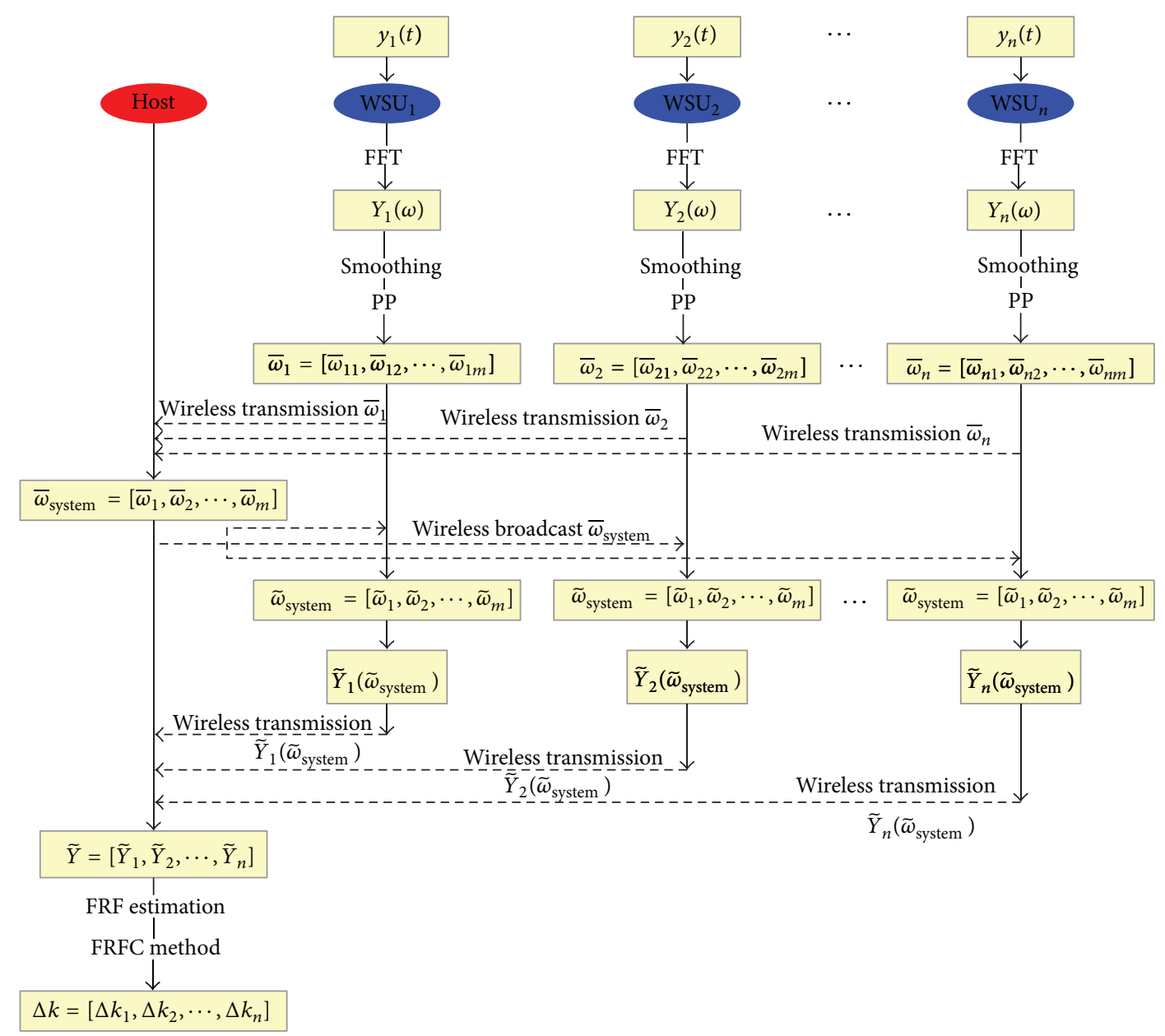

FIGURE 18: Implementation of the FRFCM in a wireless sensing system [77].

an embedded FFT algorithm and a set of eigenfrequencies of the structure in the $i$ th WSU is determined by the embedded smoothing algorithm and PP algorithm. The frequency set selected in each WSU is then transmitted wirelessly to the host computer. The most probable set of the system eigenfrequencies is decided by taking the median of each set of peaks in the host computer and then broadcasted to all the $n$ WSUs. The $i$ th WSU then transmits a set of Fourier spectra around the system's eigenfrequencies back to the host computer. After the host computer receives the selected frequency spectrum segments from all the WSUs, the FRF segments are estimated by dividing the cross-power spectrum between the structural response and input excitation. With the system matrices and FRFs of the structure in the reference state written in the host computer, the variations of stiffness matrices can be calculated. The procedure of implementation of the FRFCM on a wireless sensing system is displayed in Figure 18. The laboratory test results demonstrate that, by taking advantage of collocated computing resources in wireless sensors, the proposed FRFCM can locate and quantify damage with acceptable accuracy and moderate energy efficiency.

Kim and Lynch [78] have proposed a system identification strategy for subspace system identification based on Markov parameters (MPs). The method is specifically customized for embedment within the decentralized computational framework of a wireless sensor, Narada, proposed by Swartz et al. [35]. First, individual wireless sensor nodes perform local data processing to identify the MPs of a structural system. Then, the estimated MPs are wirelessly communicated to a central server where the global structural properties are assembled by execution of the Eigensystem realization algorithm (ERA). The difference of the modal characteristics autonomously extracted by the in-network data processing was found to be within $2 \%$ of those extracted offline by subspace identification for the first four models.

Yun et al. [79] have suggested a RemoteSensingDP module to implement decentralized damage identification in Imote2. The RemoteSensingDP module conducts data analysis capability using wavelet transformation and is written by the nesC language. The wavelet used in this implementation is the Haar wavelet. The onboard microprocessors conduct wavelet transformations to compute wavelet coefficients corresponding to the accelerations. The wavelet coefficients are transmitted wirelessly to the central server, which is connected to the laptop computer. Then the wavelet energy and entropy can be computed. Finally, the damage can be 
identified through wavelet entropy indices. The proposed method was conducted using a bench-scale three-story shear building and a three-dimensional truss bridge structure in a laboratory. It was verified that the damage identification method with wireless sensor nodes could successfully locate damage in a laboratory benchmark truss structure.

The Hilbert-Huang transform (HHT) is an adaptive signal processing technique that produces signal decomposition in both time and frequency domains, providing instantaneous frequencies, phase, and damping for extracting damage sensitive features from the processed and decomposed data. Zhang [80] proposed a new distributed SHM scheme based on HHT for the implementation in the intrinsically decentralized computing environment in WSNs. The HHT-based decentralized data processing approach has been verified with both simulation and experiment. The results show that the proposed method achieves higher accuracy for identifying modal characteristics. The onboard computing features of the proposed system are also proven to be tolerant towards environmental fluctuations in temperature and noise levels.

In the independent processing, measured data are processed by each sensor node independently without communicating with other sensor nodes. After processing, the amount of data wirelessly transferred in the network is significantly reduced. Hence, these embedded data processing methods are relatively power efficient when compared to the transfer of raw data to a central server. However, there is no sharing of sensor data (raw or processed) between nodes, preventing these centralized algorithms from autonomously producing system-wide spatial properties (e.g., model shapes). Additionally, because of their inherently centralized communication architecture, these methods are not scalable to large or spatially diverse sensor networks $[37,81]$.

3.3.3. Coordinated Processing. In the coordinated computing strategy (Figure 17(c)), a wireless sensor processes data by a local computational core and exchanges information with other wireless sensors by wireless transmission, which is different from the independent processing. Through cooperation among different wireless sensors, more complicated data processing strategies and damage diagnosis approaches can be realized. Short data transmission distance and highly compressed data volume make the coordinated computing strategy more power efficient. The execution of damage diagnosis using WSNs is more automatic. WSNs employ a coordinated computing strategy to offer a scalable solution that has the potential to dramatically improve SHM efforts. But the coordinated computing strategy requires a more complex network topology, which makes the management of the network difficult.

The most significant contributions in this direction are the one presented by Gao et al. [58] with the introduction of the distributed computing strategy (DCS). Small numbers of wireless sensors are grouped to form different communities so that all sensors in a community are located in the range of a single hop. Although each sensor in Figure 17(c) is included in only one community, in the DCS approach, wireless sensors can be contained in multiple communities. Each cluster-head collects necessary information and implements the damage detection algorithm for its community. Adjacent cluster-heads interact with each other to exchange information. Once information is aggregated, the clusterheads determine the information to be sent back to the central server. This coordinated processing allows the sensor nodes to communicate with one another and share information in order to be able to keep spatial information that can be used to produce a global picture of a bridge system. The cluster-head of the community in which damage has not occurred transmits only an "OK" signal to the central server. If damage has occurred, the cluster-head of the community needs to send damage information such as damage location. Thus, the DCS approach requires only limited information which needs to be transferred between sensors throughout the entire sensor network. This approach will significantly reduce the communication traffic in the sensor network. The natural excitation technique (NExT), ERA, and damage locating vector (DLV) method can be utilized as structural analysis techniques to materialize this DCS concept [21].

To verify the DCS concept, Gao and Spencer Jr. [82] have proposed a decentralized flexibility-based damage detection method by extending the DLV method [83]. By utilizing only locally measured information, the damage of the local elements within these communities is detected. The damage detection results in these communities are then communicated with the surrounding communities and sent back to a central server through each community's cluster-head. Continuous online monitoring of a structure can be done without relying on central data processing. This approach will significantly reduce the communication traffic and the associated power demands in the sensor network. Numerical simulation demonstrated that the proposed approach works well for both single and multiple damage scenarios. The experimental results have shown that the proposed DCS approach can successfully monitor local community members only utilizing locally measured information for various damage scenarios [84]. The proposed DCS approach was shown to be promising for application of SHM in a densely distributed sensor network.

Spencer Jr. et al. [21, 85, 86] further established a framework for SHM on the WSN by using DCS. After the first measurement, the cluster-head broadcasts its time history to all the leaf nodes in the community. On receiving the record, each leaf node calculates the spectral density between its own data and the received record. This spectral density estimate is locally stored. After each measurement, the nodes repeat this procedure one time and the stored value is updated by taking a weighted average between the stored value and the current estimate. In this way, cross-spectral density is calculated on each node, and the estimated spectral densities are converted to correlation functions by inverse FFT and sent back to the cluster-head. Subsequently, modal analysis is conducted independently in each sensor group to estimate the local modal information using the ERA or the NExT/ERA. After that, local model shapes are assembled using a least squares approximation to estimate the global modal properties in the base station. Finally, the DLV method or stochastic damage 


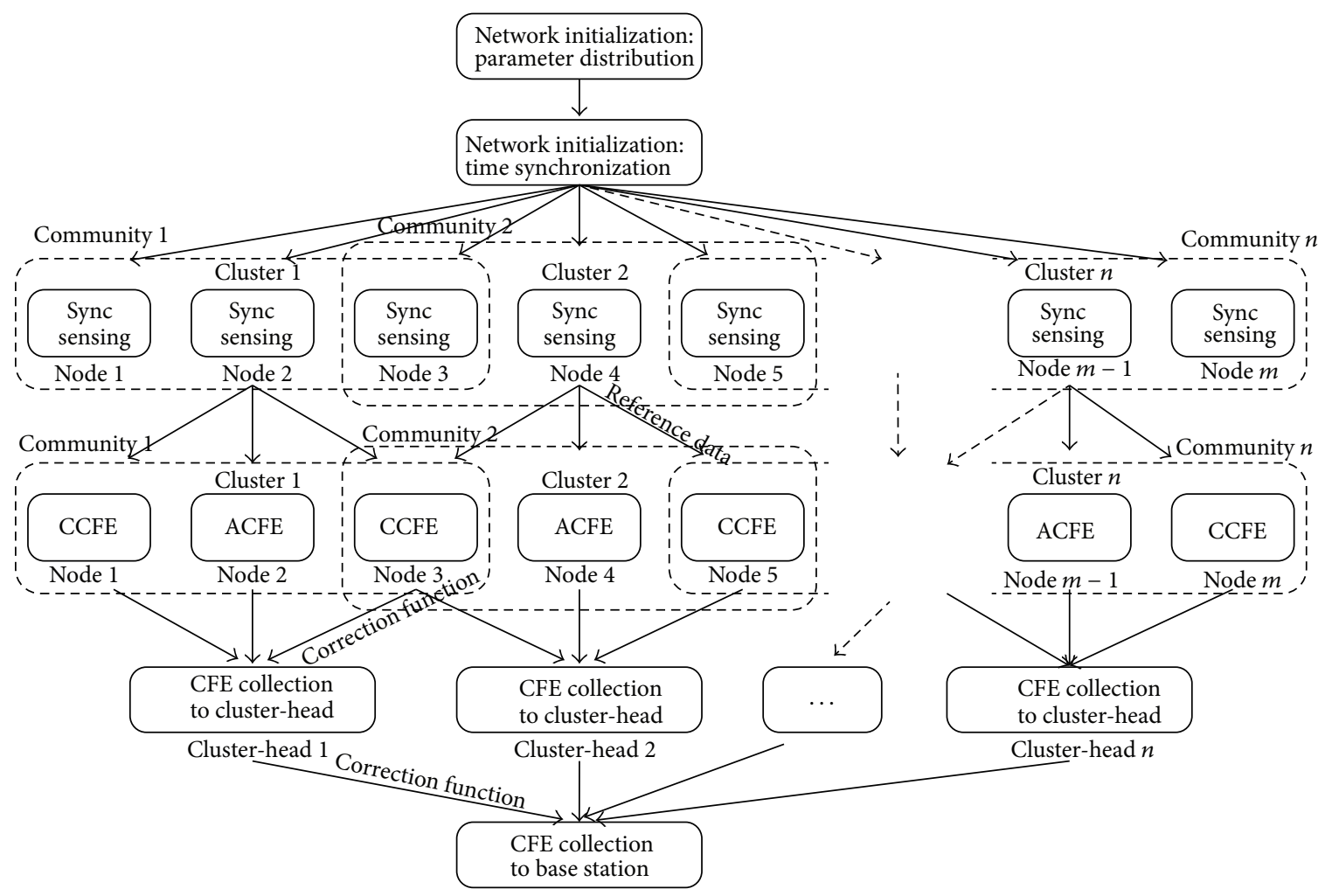

ACFE: auto-correlation function estimation.

CCFE: cross-correlation function estimation.

FIGURE 19: Information flow for NExT-based coordinated processing [46].

locating vector (SDLV) method [87] is used to localize the damaged element using modal properties identified before and after damage. Figure 19 shows the information flow in the NExT-based coordinated processing scheme. The method is experimentally verified on a steel truss structure using a small number of SHM-H sensor boards as cluster-heads and the SHMA board for the leaf nodes in the sensor network. The results showed that the proposed approach for decentralized modal analysis and damage diagnosis is efficient [46], while the ERA and NExT/ERA can be replaced by other system identification methods such as stochastic subspace identification (SSI) [60] or frequency domain decomposition (FDD) with random decrement technique (RDT) [80]. A similar framework was also employed by Yan et al. [88, 89]. In their method, modal identification is achieved using the FDD method and the PP technique.

Based on the hybrid SHM method proposed by Kim et al. [90], a modified global and local damage detection scheme was designed by Park et al. [43] to monitor prestressed concrete (PSC) bridges. The basic idea of the scheme is that the vibration-based SHM methods that are embedded in the acceleration-based sensor nodes (i.e., Acc-SSNs) are used for alarming damage occurrence at a global structure level and, at the same moment, the impedance-based SHM method that is embedded in the impedance-based sensor nodes (i.e., Imp-SSNs) is used for pinpointing damage occurrence at a local member level. The displacement ratio in the frequency domain (i.e., frequency-response ratio) between two outputs at different locations is used as the global damage alarming indicator, while the root-mean-square deviation of impedance signatures measured before and after damage is used to quantify the local damage in the structure. From the experimental evaluation, the wireless sensor nodes accurately alarmed the occurrence of damage in the tendon and girder of the PSC girder model.

3.3.4. Parallel Processing. Zimmerman et al. [36] have proposed a distributed data processing strategy, which is named parallelizing processing. The sketch of data flow is described in Figure 17(d). They have adopted and modified three distributed output-only modal identification techniques: the PP method, the FDD method, and the RD method. The first one is the PP method. First, acceleration time-history data is collected at each sensor node and converted to an FRF using an embedded FFT algorithm. Then, each node picks the largest peaks from its FRF. Finally, the central server in the WSN can infer a subset of reasonable modal frequencies from the original PP data provided by wireless sensors. This method is relatively simple to implement in a sensing network. However, PP is always difficult to implement perfectly in a software and does not properly handle closely spaced modes. The second one is the FDD technique. At first, modal frequencies at each node are identified. Then, 


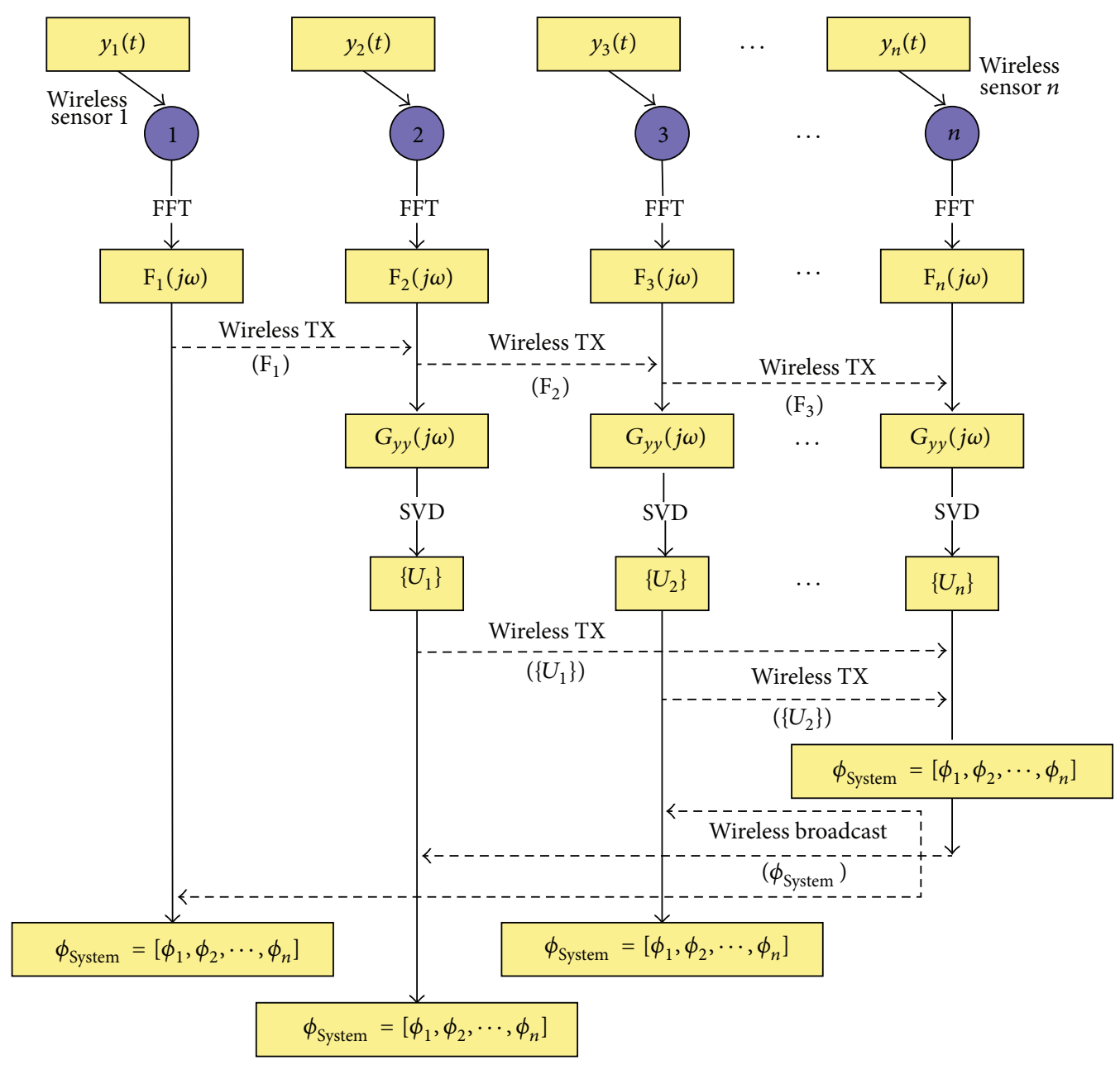

FIGURE 20: Implementation of the FDD method in a network of wireless sensors [36].

every unit in the network transmits its complex FFT results to the next node in a predetermined chain to construct a two-degree-of-freedom PSD matrix. After performing SVD on all of the PSD matrices, a set of two-node model shapes can be extracted at each natural frequency. Finally, all of the two-node model shapes are transmitted to the central server, where they are combined to form the global mode shapes of the structural system. Figure 20 presents the implementation of the FDD method in a network of wireless sensors. Because all FFT and SVD computations are performed simultaneously in a parallel fashion, significant time saving and effective data reduction can be realized. The FDD provides more reliable and robust model shape estimation compared to $\mathrm{PP}$, while this approach requires a linear network topology and may result in substantial accumulation of errors in the global mode shapes. Subsequently, a market-based frequency domain decomposition (MBFDD) technique derived from free-market principles is developed by Zimmerman and Lynch [91] through which an agentbased WSN can autonomously and optimally shift emphasis between improving the accuracy of its mode shape calculations and reducing its dependency on any of the physical limitations of a wireless network, namely, processing time, storage capacity, wireless bandwidth, or power consumption. In contrast to the FDD method, which uses a predefined chain-like topology through which computational tasks are distributed, the MBFDD technique creates an ad hoc treelike topology through which a set of SVD calculations of varying sizes can be distributed. Numerical results show that the MBFDD is capable of autonomously forming a computational topology that allows a network of wireless sensors to not only improve upon the mode shape estimates of the FDD technique but also optimally distinguish between multiple resource constraints or objectives. The third one is the RD method. Firstly, a set of consistent time-history acceleration data is collected at each sensing node and transfered to the frequency domain. Then, the SDOF free decay impulse response functions are obtained by applying window filter and embedded IFFT. Finally, zero crossing and logarithmic decrement techniques are employed to automatically extract modal frequency and damping information from the impulse response. This method provides accurate estimates of modal damping ratios by taking advantage of the great degree of redundancy available within a sensing network. However, in a multiple-degree-of-freedom system, prior knowledge of the frequency characteristics of the system is required in order to 
properly window the Fourier spectrum. This method is also not suited to determining modal properties involving closely spaced modes.

Zimmerman and Lynch [37] have presented novel wireless parallel simulated annealing (WPSA) to update structural models. In the WPSA, a computational task requiring simulated annealing (SA) optimization is first assigned to any one available sensing unit, along with a user-defined initial temperature, $T_{0}$. This first wireless sensor, $n_{0}$, then beacons the network, searching for other sensors available for data processing. If a second sensing node, $n_{1}$, is found, the first sensor, $n_{0}$, will assign the SA search tree starting at the next temperature step, $T_{1}$, to the second sensor, $n_{1}$, passing along its current information regarding the most optimal system state yet visited. This process continues until no sensors remain available for data processing.

3.4. Power Management. Though self-powering of wireless sensors avoids the effect of the AC power-line network on composition of the sensor network and electric surge in the outdoor use, it also causes one of the major challenges in WSNs, sustainable power supply. Wireless sensors typically are battery-powered, and their capacity is still limited although many high capacity batteries were invented like the lithium battery. Once the battery has consumed all of its power, replacement of the battery located remotely can become a very expensive and tedious or even impossible task. Power management is one of the biggest considerations when a large-scale WSN for bridge health monitoring is deployed. There are three relative ways to manage power and prolong WSNs lifetime. The first one is selecting low power consumption hardware. The second one is using power efficiently, termed as power efficiency. And the third one is power harvest from a local place.

3.4.1. Low Power Consumption Hardware. All wireless sensor designers take low power consumption as the most important parameter to be considered when selecting wireless sensor components. With the development of the MEMS technology, the power consumption of wireless sensor components is reduced to a great extent. For example, the microcontroller AT90\$8515 consumes $40 \mathrm{~mW}$ of power [30], the X-scale processor (PXA27x) consumes $44 \mathrm{~mW}$ at a clock rate of $13 \mathrm{MHz}$ [44], the Chipcon CC2420 only consumes $60 \mathrm{~mW}$ when receiving and $52 \mathrm{~mW}$ when transmitting within the range of 10-75 $\mathrm{m}$ [92], and the MaxStream 9XCite wireless modem consumes $250 \mathrm{~mW}$ when transmitting, $150 \mathrm{~mW}$ when receiving, and less than $5 \mathrm{~mW}$ when idle [32]. But a component with low power consumption also implies poor performance. Taking the tradeoff between power consumption and requirement of SHM into account, the power consumption wireless sensor components cannot be decreased illimitably. As a result, the lifetime of the wireless sensor is still very short when comparing with the service life of a bridge. A more effective power strategy is required.

3.4.2. Power Efficiency. Power efficiency is defined as appropriately utilizing limited battery power to maximize lifetime of WSNs and to achieve the goal of bridge health monitoring at the same time. Three methods are employed generally to consume power efficiently: sleep mode, network optimization, and data compression.

Sleep mode means that most of the components on a wireless sensor are asleep and only the radio transceiver is awake to listen for beacon signals if no monitoring actions are carried out, so the power consumption can be reduced. A lot of components used for wireless sensors are produced with a sleep model, a deep-sleep mode, or different work conditions with different power consumption. Galbreath et al. [93] proposed two low-power monitoring modes. In the first mode, the microcontroller periodically awakes via a watchdog timer interrupt, turns on the telemetry hardware, and listens for a wake command from the central server. In the second mode, the microcontroller remains in the low-power sleep mode until a rising external analog voltage triggers a hardware interrupt. Rice et al. [60] designed two power-efficient management services for sensor nodes, called SnoozeAlarm and ThresholdSentry. Using the property that the Imote 2 allows the processor to be put into a deepsleep mode with minimal power consumption by making only the processor clock awake, the SnoozeAlarm service places the sensor nodes in the deep-sleep state, waking them periodically for a brief time (e.g., $0.6 \mathrm{sec}$ for the single hop and $0.75 \mathrm{sec}$ for multi-hop) to listen for beacon signals sent from the gateway node. Once a signal is heard, the node becomes fully active. The ThresholdSentry application runs on the designated sentry nodes periodically executing the sentry's task, that is, collecting data for a given period of time and checking if the threshold value is exceeded. If this threshold is exceeded, the sentry nodes send notification to the gateway node, then the gateway node wakes up the entire network and initiates designated network-wide tasks. But the current implementation of ThresholdSentry allows the network only to capture the occurrence of longer-duration, lower-frequency events such as high wind. Besides, the dutycycle approach, which wakes wireless sensors for a short time in every predetermined period, is a promising method. Whelan and Janoyan [48] stated that, for active sampling at a rate of one minute per hour, the estimated service life of their wireless senor would be extended to 160 days. Hu et al. [13] demonstrated that the service life of S-Mote is extended to 168 days if the sensor node participates in active sampling for only one hour/day.

Network optimization means finding the optimal node locations or routing so that the power consumption is minimal while the requirement of bridge health monitoring is fulfilled. Zimmerman et al. [94] have developed a resource distribution framework based on a market-based method, which is used to autonomously distribute these scarce network resources (CPU time, wireless bandwidth, storage capacity, battery power, etc.) across various computational tasks with competing objectives and/or resource demands. In this framework, a Pareto-optimal allocation of scarce resources can be reached while completing a set of multiple computational objectives as quickly as possible. And this optimization method can be expanded to other decentralized data processing strategies. $\mathrm{Fu}$ et al. [95] have considered 
the WSNs deployment problem for finding node locations to reliably diagnose the health of a structure while consuming minimum energy during data collection. A min-max, energybalanced routing tree and an optimal grid separation formulation that minimize the energy consumption as well as provide fine grain measurements are proposed.

Data compression reduces the volume of transmitted data and shortens the data transmission distance through innetwork data processing rather than delivering raw collected data, so that the lifetime of WSNs can be prolonged. Almost all distributed data processing technologies as mentioned in Section 3.3 can compress the volume of transmitted data and alleviate power consumption to some extent. For example, performing the damage detection using the AR-ARX model at the sensor node as compared to wirelessly transmitting the original raw time-history data is shown to be on the order of $50 \%$ of data compression [62-64]; the total data to be transmitted when using the ERA to implement modal analysis in the distributed data processing scheme is $N \times$ $n_{d}+N / 2 \times\left(n_{s}-1\right)$ compared to $N \times n_{d} \times\left(n_{s}-1\right)$ in the centralized data collection scheme, where $N$ is data length of each time history, $n_{d}$ is repeated times of spectral density estimation, and $n_{s}$ is the number of nodes in the community [22]; over $77 \%$ compression was accomplished using local calculation by extracting 105 MPs from 1,200-point response time-history records at 15 wireless sensor nodes [78].

3.4.3. Power Harvest. Though the battery life of a wireless sensor can be extended by efficient power management, data condensation, and in-network processing as mentioned above, the use of ordinary batteries still requires regular battery replacement. Power harvest from a local place is a rational choice. Power harvest is the process of acquiring the energy surrounding a system and converting it into usable electrical energy [96]. Recently, a surge of power harvesting researches has been brought on by the advances in wireless technology and low-power electronics. Thus, many researchers in the SHM and sensing network community have shown interest in power harvesting and have proposed alternative power sources such as sunlight, wind, and vibration [97]. Power harvest can be further classified into three categories: wireless energy transfer, ambient energy harvest, and vibration-based energy harvest. Among them, the harvesting of ambient energy and vibration-based energy is the strongest candidate for application to the bridges.

Wireless energy transfer means power is transmitted wirelessly. Mascarenas et al. [11, 98], for the first time, used wireless RF transmission to deliver electrical energy to power a piezoelectric impedance sensor node by charging the capacitor in the node. A controlled helicopter, which can fly to the wireless senor node, is used as a central server to send energy to wireless sensors and receive data from wireless sensors. The results showed good performance by transmitting necessary power to the impedance sensor node, and it took about 200 seconds to reach up to the operation voltage level $(3.3 \mathrm{~V})$ of the node. However, one of the limitations in microwave transmission is the attenuation of the wave as it travels through space. The loss associated with this attenuation has been characterized as the square of the distance between the transmitting and receiving antennas. Park et al. [99] proposed an optical system for wireless power and data transmission. It took advantage of optoelectronics for both guided wave generation and sensing. A generated waveform by modulation of a laser is wirelessly transmitted to a photodiode connected to a PZT on the structures. Then, the photodiode converts the light into an electrical signal and excites the PZT and the structure. Finally, the reflected response signal received at the same PZT is reconverted into a laser, which is wirelessly transmitted back to another photodiode located in the data acquisition unit for damage diagnosis. Since the laser emits highly directional and collimated radiation with a low angle of divergence, the energy carried by the laser beam can be transmitted for a long distance without attenuation and focused onto a small area [98].

The main sources of ambient energy are sunlight, thermal gradient, human motion, acoustic noise, and so forth. Multiple articles reviewing the prospects of ambient energy sources for harvesting can be found in the literature [100104]. From the practical reviews of SHM application, sun energy is perhaps one of the most powerful choices. Raghunathan et al. [105] systematically analyzed the components, design choices, and tradeoffs involved in designing a solar energy harvesting module. The proposed module allows for near-perpetual operation of the Mica2 mote. Jang et al. [106] successfully carried out the validation of solar energy harvesting in the Jindo Bridge deployment using solar panels. The installed solar panel is plotted in Figure 21(a). Chae et al. [9] used a solar cell and rechargeable battery to secure a stable power supply of the WSNs deployed on the Yongjong Grand Bridge. Another promising energy source is wind power in long-span bridges, which are usually located in windy areas. Spencer Jr. and Cho investigated the feasibility of small wind-powered generators to operate wireless sensors located at a cable-stayed bridge to show the maximum output power of a wind turbine is enough [2]. Anant Kishore and Priya [107] provided a $40 \mathrm{~cm}$ diameter small wind energy portable turbine (SWEPT), which has rated the power output of $1 \mathrm{~W}$ with the rated wind speed of $4.0 \mathrm{~m} / \mathrm{s}$ and is capable of producing power output up to $2.2 \mathrm{~W}$ at the wind speed of $5.5 \mathrm{~m} / \mathrm{s}$. Figure 21(b) shows the prototype of SWEPT.

Vibration-based energy harvest represents another important power harvest technology. The most effective method of implementation of vibration-based energy harvest is the use of piezoelectric materials [108]. Piezoelectric materials have a crystalline structure that provides them with the ability to transform mechanical strain energy into electrical charge. This property provides these materials with the ability to transfer ambient vibration into electrical energy that may be stored and used to power other devices. The bridges on which wireless sensors are installed are vibrated continuously, which provides a natural source of vibration-based energy. The concept of utilizing piezoelectric materials for energy generation has been studied by many researchers over the past few decades. It was already developed as an independent subject. Overviews, discussions and comparison of vibrationbased energy harvest have recently been given by many 


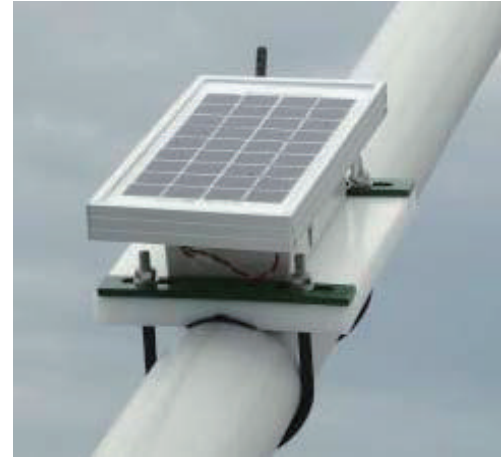

(a) Solar panel [2]

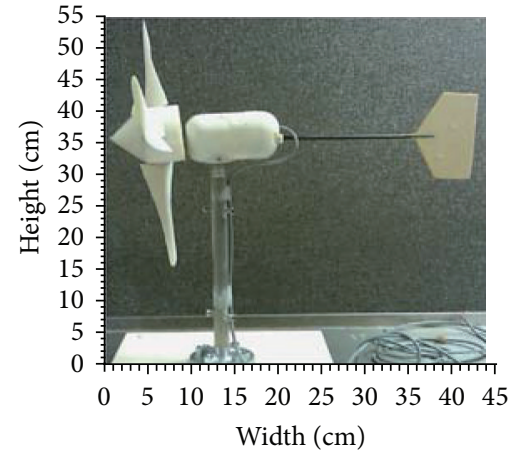

(b) SWEPT prototype [107]

Figure 21: Ambient energy harvest.

literatures which include, but are not limited to, Sodano et al. [96], Dutoit et al. [109], Park et al. [110], Gilbert and Balouchi [111], Khaligh et al. [112], and Kim et al. [113]. Because vibration-based energy harvesters are still in the development stage, only a few commercial solutions are available. Most research efforts are still in proof-of-concept demonstrations in a laboratory setting.

3.5. Time Synchronization. Due to the delay of radio transmission or inherent internal sensor clock errors, the collected dates in different wireless sensors in WSNs may initially be unsynchronized. A time synchronization (TS) error in a wireless sensor network can cause inaccuracy in bridge health monitoring applications. The effect of the TS error of the nodes on the accuracy of modal analysis is analyzed by Krishnamurthy et al. The paper shows that a relatively small error in TS (e.g., $30 \mu \mathrm{s})$ can cause a considerable reduction in the reliability of the mode shape reconstruction of higher modes, which are normally better indicators of structural damage. It is concluded that the effect of the TS error is comparable or can even exceed the effect of sensor's noise $[20,22]$. Since the appearance of WSNs, TS has been one of the most addressed issues. To data, many TS protocols have been developed. For more detailed information on the TS, it is recommended to refer to those papers by Sivrikaya and Yener [114], Rhee et al. [115], Lasassmeh and Conrad [116], Ranganathan and Nygard [117], and Yang [118], where more than 60 references are cited. Two of the most notable TS protocols are the flooding time synchronization protocol (FTSP) proposed by Maróti et al. [119] and the timing-sync protocol for sensor networks (TPSN) proposed by Ganeriwal et al. [120].

FTSP performs both offset adjustment and estimation of the relative skew of the clocks through a one-way message exchange, decreasing the frequency of transmission of the required TS beacons and hence energy consumption. The FTSP was implemented by Nagayama and Spencer Jr. in the Imote2 [85]. FTSP utilizes time stamps on the senders and receivers. When a beacon node broadcasts a packet to the other nodes, a time stamp, $t_{\text {send }}$, is appended at the end of the packet. Upon reception of the packet, the receivers stamp the time, $t_{\text {receive, }}$, from their own local clocks. The delivery time, $t_{\text {delivery }}$, between these two events is usually not small enough to be ignored, but the variance of $t_{\text {delivery }}$ over time is usually small. The offset between the local clock on the receiver and the reference clock on the sender is determined as $t_{\text {receive }}-t_{\text {send }}-t_{\text {delivery. This offset is subtracted from the }}$ local time when global time stamps are requested afterward. A group of nine Imote2s was experimented and a TS error of $10 \mu \mathrm{sec}$ is achieved, which is small for SHM applications.

TPSN periodically adjusts the offset of the clocks of the nodes using a two-way message exchange. However, due to a required previous hierarchy formation phase and to the two-way message exchange, it introduces a significant communication overhead, wasting energy and radio channel resources [20]. The first step of the TPSN protocol is to create a hierarchical topology in the network. Each node is assigned a level in this hierarchical structure. A node belonging to level $i$ can communicate with at least one node belonging to level $i-1$. Only one node is assigned level 0 . Once the hierarchical tree structure is established, a node with level $i$ synchronizes to a node with level $i-1$. After all, every node is synchronized to the root node with level 0 and TPSN achieves networkwide time synchronization [114]. Mica2 motes employing TPSN are reported to synchronize with each other to an accuracy of $50 \mu \mathrm{sec}$ [21].

\section{Performance Validation and Application}

4.1. Scaled Bridge Test. Performance validation within the well-controlled laboratory environment is the first step towards deployment of WSNs-based bridge health monitoring systems on field bridges. The earlier efforts are bonding several wireless sensors on shaking table or rotator with predetermined input signals; the accuracy and reliability of those wireless sensors are assessed. Those works have been finished after wireless sensors had been designed, which are relatively easy and are not reviewed in this paper. However, more recent studies have begun to deploy complicated WSNs-based bridge health monitoring systems on partial- and full-scale 
bridge models, which provide comprehensive insight into the opportunities and challenges of the WSN technologies for bridge monitoring.

Whelan et al. [121] have applied a wireless sensor network platform to perform system identification on a bridge model throughout healthy and damage case states in an effort to assess the performance of the Wireless Sensor Solution developed at Clarkson University $[122,123]$. The laboratory bridge features a four-meter span, one-meter wide, steel frame with a steel and cement board deck composed of sheet layers to regulate mass loading and simulate deck wear. A rectangular measurement grid was developed across the steel frame of the bridge model resulting in twenty-eight measurement locations. A wireless node interfaces with a dualaxis LIS2L02AL accelerometer at each measurement location for a total of 56 channels of wireless measurement. These wireless channels are accommodated by two WSNs operating at adjacent carrier frequencies that are synchronously triggered by an external switch wired to interrupt signals at the central network coordinator nodes. Comparison between the acceleration measurement transmitted by the wireless sensor node and the cable-based sensor located at the same place reveals exceptional correlation. The power spectrums contain nearly identical frequency content and magnitudes, thereby validating the transfer function of the wireless sensor signal conditioner and indicating comparable performance between the two data acquisition systems.

Park et al. [43] have verified the proposed autonomous operation schemes using the Acc-SSNs and Imp-SSNs installed on a lab-scaled prestressed concrete girder model. The girder with a T-beam section has the span length of $6 \mathrm{~m}$. Seven Acc-SSNs and seven conventional accelerometers were located on the two symmetrical sides of the test structure. The Imp-SSN was placed on the anchor plate. The impact excitation was applied by an electromagnetic shaker (VTS100) at a location distanced at $1.7 \mathrm{~m}$ from the right edge. The vibration-based SHM methods were embedded in the AccSSNs and the impedance-based SHM method was embedded in the Imp-SSNs. Frequency responses acquired from the Acc-SSNs and the conventional system show good matches with little difference. Those two impedance signals measured by the Imp-SSN and the commercial analyzer show a great agreement.

Bocca et al. [20] attached eight ISMO-2 nodes to a wooden model bridge to evaluate the capability of the ISMO2 nodes and proposed a time-synchronized wireless SHM system. The bridge is $420 \mathrm{~cm}$ long, $65 \mathrm{~cm}$ wide, and $34 \mathrm{~cm}$ high, as shown in Figure 22. Sixteen wired accelerometers were also used as reference. The locations of the wireless sensors and wired sensors are plotted in Figure 22. The singlehop star topology was employed to establish the network. All along the tests, the sampling frequency of the wired sensors was set to $1,024 \mathrm{~Hz}$. The sampling frequency of the ISMO-2 nodes was varied, from a minimum of $200 \mathrm{~Hz}$ to a maximum of $1 \mathrm{kHz}$. The measurement period was initially set to 40 seconds and then progressively increased to 2 minutes. Also, different 1-axis, 2-axis, and 3-axis measurement sets were collected. During the tests, the achieved final packets delivery ratio was $99.95 \%$. The modal properties identified

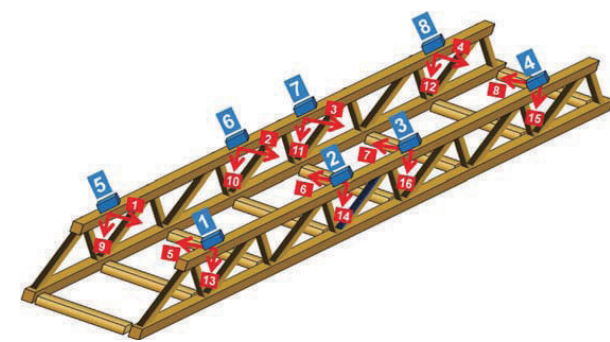

FIgURE 22: The sensors deployed on the wooden model bridge: 16 wired accelerometers (red arrows), 8 ISMO-2 nodes (blue boxes) [20].

with acceleration signals collected by wired sensors and by the wireless nodes have shown $1.035 \%$ maximum relative difference.

4.2. Full-Scale Bridge Test. Although the functionality and the stability of many wireless sensors and WSN prototypes have been thoroughly verified by a series of experiments at the laboratory scale bridges, validation of field performance must be done using actual civil bridges for the reason that the noise floor, data transmission distance, network topology, signal interference, and so forth in the practical environment has much difference with those in the laboratory environment. Another attractive benefit is that the WSNs can be further used for the bridge monitoring once their reliability is validated.

Before 2005, only a handful of nodes are instrumented on the actual bridges. Only the feasibility of monitoring the structural response using wireless sensors is validated. Aoki et al. [124] installed only one wireless accelerator on the Tokyo Rainbow Bridge to test the performance of the developed MEMS-based wireless sensor. Galbreath et al. [93] demonstrated the use of a wireless sensor network to monitor the performance of a steel girder composite deck highway bridge. Binns [125] has presented a field validation study of a wireless monitoring system on a highway bridge in Ohio. Lynch et al. [30] deployed seven wireless sensing units interfaced with MEMS-based capacitive accelerometers on the Alamosa Canyon Bridge to validate the performance of the wireless sensing unit in the field. Chung et al. [126] have installed a network of wireless sensors upon a footbridge on the University of California, Irvine, campus to monitor bridge responses under footfall excitation.

In 2006, Heo et al. [39] deployed the improved SWMAS on a self-anchored suspension bridge in Korea to assess the function of the new SWMAS in use of a real flexible structure. The main span and the side span of the bridge are $300 \mathrm{~m}$ and $125 \mathrm{~m}$, respectively. Its truss structures are the two-storied Warren trusses. The bridge sustains compressive force and upper eccentric force with its stiffening trusses. The improved SWMAS devices were installed on the hanger rope and at the center of the main span to measure how the bridge reacts to the ambient vibration caused by traffic and wind load. The sampling rate of the wireless sensor unit is set at $80 \mathrm{~Hz}$ and the collecting lasted for 100 seconds. The tensile force calculated from the hanger rope differed from the office data by $6.7 \%$. 
The main span's natural frequencies remained within a 3\% range. The usefulness of the SWMAS for monitoring flexible structure systems was proved.

To quantify the accuracy and robustness of wireless monitoring systems within the complex environment encountered in the field, Lynch et al. $[127,128]$ installed a network of lowcost wireless sensors in the Geumdang Bridge, Korea. In total, 14 wireless sensors were installed in the concrete box girder span to record acceleration responses of ambient and forced vibrations, and the single-hop network was used for data transmission. The low-cost PCB Piezotronics 3801D1FB3G MEMS capacitive accelerometers were selected for use with the wireless monitoring system. In order to enhance the resolution of the capacitive accelerometers interfaced to the wireless sensors, a signal conditioning circuit that amplifies and filters low-level accelerometer outputs was proposed. The performance of the complete wireless monitoring system was compared to a commercial tethered monitoring system that was installed in parallel. The free-vibration response recorded by the tethered sensor and high-resolution wireless sensor indicates that they are nearly identical. The performance of the wireless monitoring system is shown to be comparable to that of the tethered counterpart. The decentralized FDD is also used to estimate the mode shapes of this bridge. The results have strong agreement with those extracted by the centralized FDD method using MATLAB. So, the effect of the decentralized FDD is validated in real circumstances.

In 2007, Kim et al. [12, 42, 129] deployed the largest WSN at that time for SHM on the Golden Gate Bridge in San Francisco. The Golden Gate Bridge is a cable-supported bridge, with a tower height of $227 \mathrm{~m}$ above sea level and a $1280 \mathrm{~m}$ long main span. 64 wireless sensor nodes, which were integrated by Crossbow MicaZ motes and new customized accelerometer boards, were distributed over the main span and the tower, collecting ambient vibrations synchronously at $1 \mathrm{kHz}$ rate, with less than $10 \mu$ s jitter, and with an accuracy of $30 \mu \mathrm{G}$. Those nodes formed a 46-hop network, with a bandwidth of $441 \mathrm{~B} / \mathrm{s}$ at the 46 th hop. To provide time synchronization in the network, FTSP is used. The recorded data are transferred from each node to the base station using multihop communication and pipelining. The data on network performance confirmed the effectiveness of spatial reuse of radio bandwidth through pipelining in maintaining a scalable network. The test results showed that the pipelining of data is very effective in providing a constant and reliable effective bandwidth for a large number of hops. Analysis of the ambient vibration data and system identification results demonstrate that high spatial and temporal sensing using the wireless sensor network give a high resolution and confidence in the identified vibration modes. Then, the collected data are used for statistical analysis of vibration modes of this bridge [130]. However, a complete cycle of sampling and data collection for the full network produces $20 \mathrm{MB}$ of data and takes about 9 hours. The efficiency of data collection in this WSN should be improved.

To assess the capabilities of a low-cost wireless structural monitoring system to rapidly collect the dynamic responses of a large-scale civil infrastructure system, Weng et al. [131] employed the rapid-to-deploy wireless sensor prototype by
Wang et al. [34] to monitor the newly retrofitted Gi-Lu cablestayed bridge (Nantou County, Taiwan) during ambient excitation conditions. Twelve wireless sensors each containing a four-channel sensor interfaced with a high-resolution ADC are used. Transducers interfaced to each wireless sensor node are highly sensitive Tokyo Sokushin VSE-15 velocity meters whose sensitivity constant is $0.25 \mathrm{~V} /$ kine (where 1 kine is equal to $1 \mathrm{~cm} / \mathrm{s}$ ). Due to the fact that only 12 wireless sensorvelocity meter pairs are available, the wireless monitoring system is reconfigured during testing to achieve three different test configurations. Data was sampled at 100 points per second on each channel to provide good waveform definition. The wireless monitoring system consists of a distributed network of wireless sensors in direct communication with a high-performance data repository where data is stored and analyzed. During data collection, the wireless monitoring system experienced no data loss as a result of the highly robust communication protocol. The measurement of structural response to ambient levels of wind and traffic has proved to be an effective means of identifying the dynamic properties of a full-scale cable-stayed bridge. Furthermore, the performance of the FDD and SSI is evaluated based on those data.

From 2009 to 2011, the Laboratory for Intelligent Infrastructure and Transportation Technologies (LIITT) at Clarkson University carried out a series of tests on four bridges using the wireless sensor system (WSS). The WSS is based on Tmote Sky mote, which conducts strain- and vibration-based monitoring through a switch. Firstly, a large-scale network consisting of 40 channels of sensor measurements acquired through 20 remote wireless transceiver nodes operating with a sampling rate of $128 \mathrm{~Hz}$ was deployed on Wright Road Bridge in St. Lawrence County, NY [48, 122, 123, 132, 133]. Dynamic behavior of the structure from ambient and traffic loads was measured. Successful acquisition of high-rate, lossless data on the highway bridge validates the proprietary wireless network protocol within an actual service environment. Secondly, the STMicroelectronics LIS2L02AL low-noise capacitance-based accelerometers were distributed across the deck surface of the Nine Wells Rail Bridge to record the ambient vibration response due to traffic loading [134]. Then, operational modal analysis was performed based on the measurement data. Thirdly, the deployment of a largescale, high-density, real-time wireless sensors network for the acquisition of local acceleration measurements across a highway bridge was executed [135]. The deployment consisted of 30 dual-axis accelerometers installed across the girders of the bridge. Output-only system identification of the structure from the experimental data is presented to provide estimates of natural frequencies, damping ratios, and operational mode shapes. Fourthly, a WSS, which is composed of 30 dual-axis LIS2L02AL MEMS accelerometers for a total of 60 channels and 30 strain transducers, was configured on the RT345 Bridge [4, 14, 136]. Modal and strain-based characteristics of the superstructure were acquired through measurements taken from those sensors over a 2-day period. That deployment demonstrates that the developed wireless sensor network has matured to the degree that modal analysis of large civil structures with a distributed network is a currently feasible and comparable alternative to cable-based 
measurement approaches. But the single-hop star topology wireless communication makes it difficult to be extended to large-scale bridges.

The hardware and software developed by Spencer Jr. et al. [2, 47, 106] have been validated through full-scale deployment on the 2 nd Jindo Bridge test-bed in Korea. The bridge is a cable-stayed bridge with a main span of $344 \mathrm{~m}$. The Jindo Bridge test-bed is constructed as an international collaborative research effort between the USA (University of Illinois at Urbana-Champaign), South Korea (KAIST and Seoul National University), and Japan (University of Tokyo). The collaborative project resulted in the first long-term and the world's largest deployment of wireless sensors to monitor civil infrastructure. The 1st deployment on the bridge test-bed was carried out in 2009. The 70 sensor nodes were installed and divided into two subnetworks: one on the Jindo island side and the other on the Haenam side. The Jindo subnetwork consists of 33 nodes ( 22 deck nodes, 3 pylon nodes, and 8 cable nodes). The Haenam subnetwork consists of 37 nodes (27 deck nodes, 3 pylon nodes, and 7 cable nodes). One base station was used to collect data for each subnet using a gateway node. Each gateway node consists of an Imote2, interface board, and antenna. Each leaf node is composed of an Imote2, battery board, sensor board, antenna, and environmentally hardened enclosure. 3D-cell batteries were used to power most of the leaf nodes; 8 nodes of the 70 were selfpowered using solar panels and rechargeable batteries. SHMA boards were used to measure acceleration, temperature, humidity, and light for most of nodes; the SHM-W sensor board was used to measure the signal from the 3D ultrasonic anemometer. The 2 nd deployment of advanced hardware and software was implemented on the test-bed bridge in 2010 . Energy harvesting strategies were employed for all sensor nodes based on the satisfactory performance during 2009; additionally, a miniwind turbine was installed on one node to assess the potential for wind energy harvesting. The network size was also increased to a total of 669 channels on 113 sensor nodes and has been divided into four subnetworks, which is currently the world largest WSN for SHM. For better understanding of wind environment, two more $3 \mathrm{D}$ ultrasonic anemometers were installed with newly developed SHM-DAQ boards. Ten SHM-H boards were implemented as cluster-heads of DCS. The wireless sensor locations are displayed in Figure 23. The estimated modal properties based on collected acceleration data are consistent with those from the wired sensor system [137]. The measured wind data are accordant well with the records of the Korea Meteorological Administration. Both results verified that the installed WSN is reliable. Successful deployment of this WSN demonstrates the suitability of the Imote 2 sensor platform, the proposed sensor board, and the developed software for full-scale, continuous, autonomous bridge health monitoring. At the same time, the tremendous potential of WSN technology for bridge health monitoring was demonstrated.

To illustrate the suitability of wireless sensors for shortterm monitoring of highway bridges, Kim et al. [138] installed a wireless monitoring system upon the Grove Street Bridge to monitor structural responses during static and dynamic load testing. A heterogeneous array of wireless sensing transducers was installed on the bridge including metal foil strain gages, accelerometers, and linear variable differential transducers. First, the acceleration response of the bridge was monitored during routine traffic loading. Modal parameters are calculated by the wireless sensors so that an analytical model of the bridge constructed in a standard commercial finite element package can be updated offline. Next, the bridge was closed to traffic and trucks of known weight were parked on the bridge to induce static deformations. The results of static load testing were used to validate the behavior of a novel jointless bridge deck constructed from a high-performance fiber reinforced cementitious composite (HPFRCC) material.

Kim et al. [139] installed a wireless sensor network on the $180 \mathrm{~m}$ long Yeondae Bridge (Korea) to measure the dynamic response of the bridge to controlled truck loading. To attain a high nodal density with a small number (20) of Narada wireless sensors, the wireless sensor network was installed three times with each installation concentrating sensors in one portion of the bridge. Intentional nodal overlapping of the three different sensor installations allows mode shapes from each installation to be stitched together into global mode shapes. The system is reconfigured with ease due to the fact that the sensors are wireless. During testing, the wireless monitoring system is found to be reliable (with no data loss encountered) and provided accurate response measurements. Using forced and free-vibration response data from the three installations, the modal properties of the bridge are accurately identified. The estimated modes are consistent with modes predicted by the finite element model.

To evaluate the potential of WSNs for use in bridge management, a network of seven sensor nodes was installed on the Ferriby Road Bridge by Hoult et al. [140, 141]. Three displacement transducer nodes were placed across cracks on the soffit of the bridge to measure the change in crack width. Three inclinometer sensor nodes were mounted on two of the elastomeric bearing pads to measure the change in inclination of the bearing pads while a final node monitored temperature in the box that contained the gateway. Due to the isolated location of the Ferriby Road Bridge, the gateway was powered by a $120 \mathrm{Ah}-12 \mathrm{~V}$ battery, which was in turn charged by a $64 \mathrm{~W}$ solar panel attached to the side of the bridge. The test results indicate that the wireless sensor networks represent a useful tool that can be used to supplement, but not replace, visual inspection. It is discovered that commercial offerings of WSNs are still geared towards research prototypes and are currently not yet mature for deployment in practical scenarios.

Kurata et al. [142-144] developed a novel wireless structural monitoring system specifically tailored for large-scale civil infrastructure systems by architecturally combining dense wireless sensor networks with a suite of information technologies remotely accessible by the Internet. The monitoring-system architecture is divided into two major layers: a lower layer consisting of low-power WSNs installed to record the environmental loading and corresponding response of the structure and an upper Internet-enabled layer offering a complete cyberinfrastructure framework for the management and interrogation of bridge response data. A wireless cellular modem is used to communicate between 


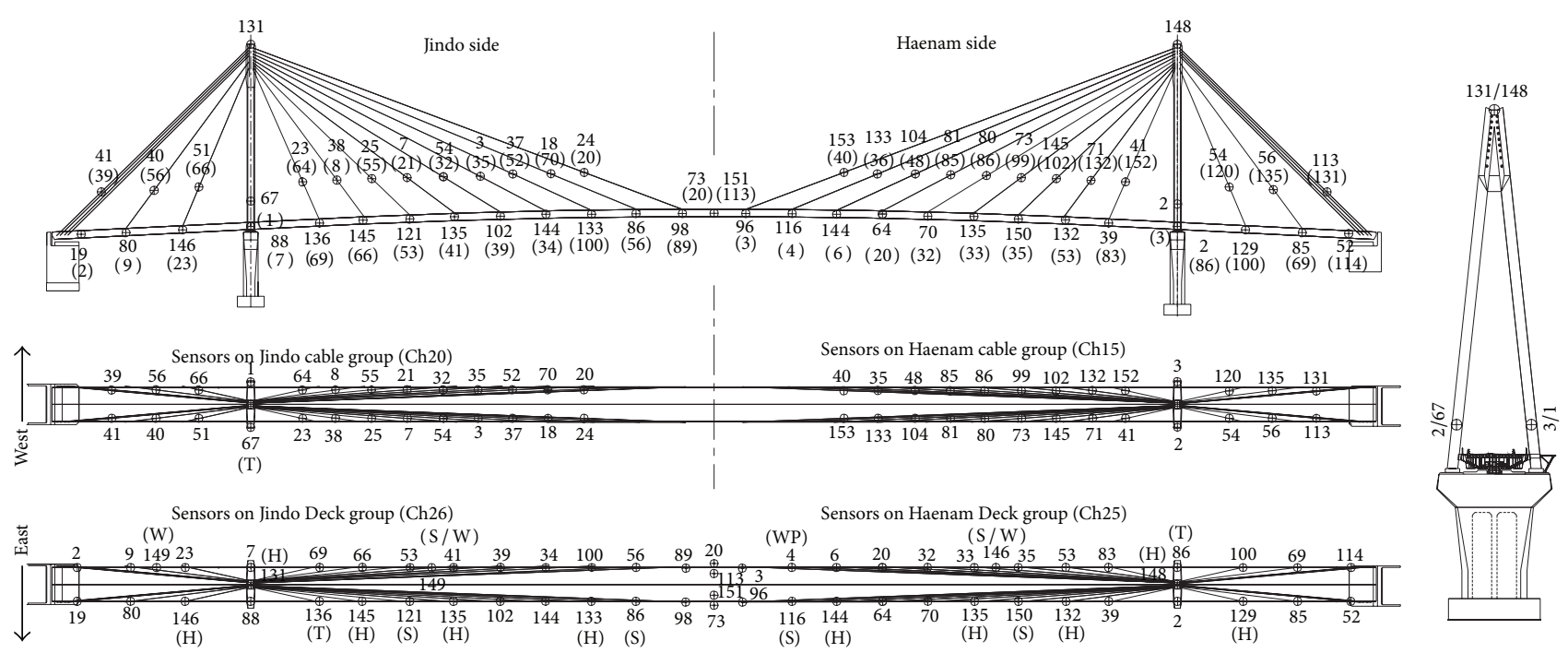

(1) ( ) values for west-side nodes

(2) $(\mathrm{H})$ means SHM-H node

(3) (S) means sentry node
(4) (W) means wind node (anemometer)

(5) (T) means external temperature node

(6) (WP) means wind power node (wind turbine)

FIGURE 23: Wireless sensor locations [2].

the WSN servers and the upper levels of the monitoringsystem architecture. To validate the long-term performance of the cyberenabled wireless structural monitoring system, the system was permanently installed on the New Carquinez Bridge (NCB). Prior to the installation of the permanent wireless system, the performance of the Narada wireless sensor nodes was evaluated on the NCB through a series of short-term deployment carried out in 2009 [145]. The permanent wireless monitoring system consists of 28 Narada nodes collecting data from 81 channels. Figure 24 presents the layout of the Narada WSN. To enhance longevity of the system, a power management strategy based on solar energy is adopted. The role of the wireless monitoring system is to drive a model-updating process to ensure that the existing finite element model of the bridge is suitable for the evaluation of the bridge immediately following a seismic event. Modal properties calculated from the data collected by the wireless monitoring system have been found to be in agreement with those extracted from the wired seismic monitoring system as well as those predicted by a high-fidelity finite element model of the bridge. The Internet-enabled wireless structural monitoring system has been proved to be scalable to a large number of nodes and has thus far proven stable and reliable over long-term use.

Kim and Lynch [146] installed a dense network of wireless sensors on the Yeondae Bridge and a multiaxle truck to identify vehicle-bridge interaction. Twenty Narada nodes, each with an integrated single-axis capacitive accelerometer (Silicon Designs SD2012), were installed permanently along the length of the bridge. The accelerometers were oriented in the vertical direction to record the vertical acceleration of the bridge. A single-axis $\pm 10 \mathrm{~g}$ accelerometer (Analog Devices ADXL105) was installed vertically at each axle and a $\pm 2 \mathrm{~g}$ accelerometer (Crossbow CXL02) was installed vertically at the center-of-gravity of the truck body. To capture the rotation of the truck body, an MEMS gyroscope (Analog Devices ADXRS624) was also installed at the center-of-gravity of the truck body. In addition, a second $\pm 2 \mathrm{~g}$ accelerometer (Crossbow CXL02) was installed in the horizontal direction at the truck center-of-gravity to measure the inertial acceleration of the truck. Each sensor installed on the truck was interfaced to a Narada wireless sensor; this allowed the truck-based sensors to freely communicate with the permanent wireless monitoring system installed on the bridge. The truck was driven across the bridge at a constant velocity with bridge and vehicle responses measured. All wireless sensor nodes use a sample rate of $100 \mathrm{~Hz}$. Time-synchronized vehicle-bridge response data is used within a two-stage system identification methodology. At first, the time-invariant properties of the bridge are estimated using the free-vibration response of the bridge. And then, the vehicle bouncing motion and the truck position history were combined with the bridge forced vibration response to estimate the time variant load matrix of the system model. Excellent system identification results were obtained with the model predicting closely the measured system response.

Chae et al. [9] deployed the ubiquitous sensor network (USN) based bridge SHM system including the u-Nodes, $\mathrm{u}$-Gateways, application software, and integrating wireless network configurations on the Yongjong Grand Bridge. ZigBee (IEEE 802.15.4) is used for short-distance communications among sensors and code division multiple access (CDMA) for long-distance wireless communications with remote locations. Five types of sensors are integrated in the system. The force balanced type accelerometer was selected to carry out the measurement of stiffening truss vibration, and 


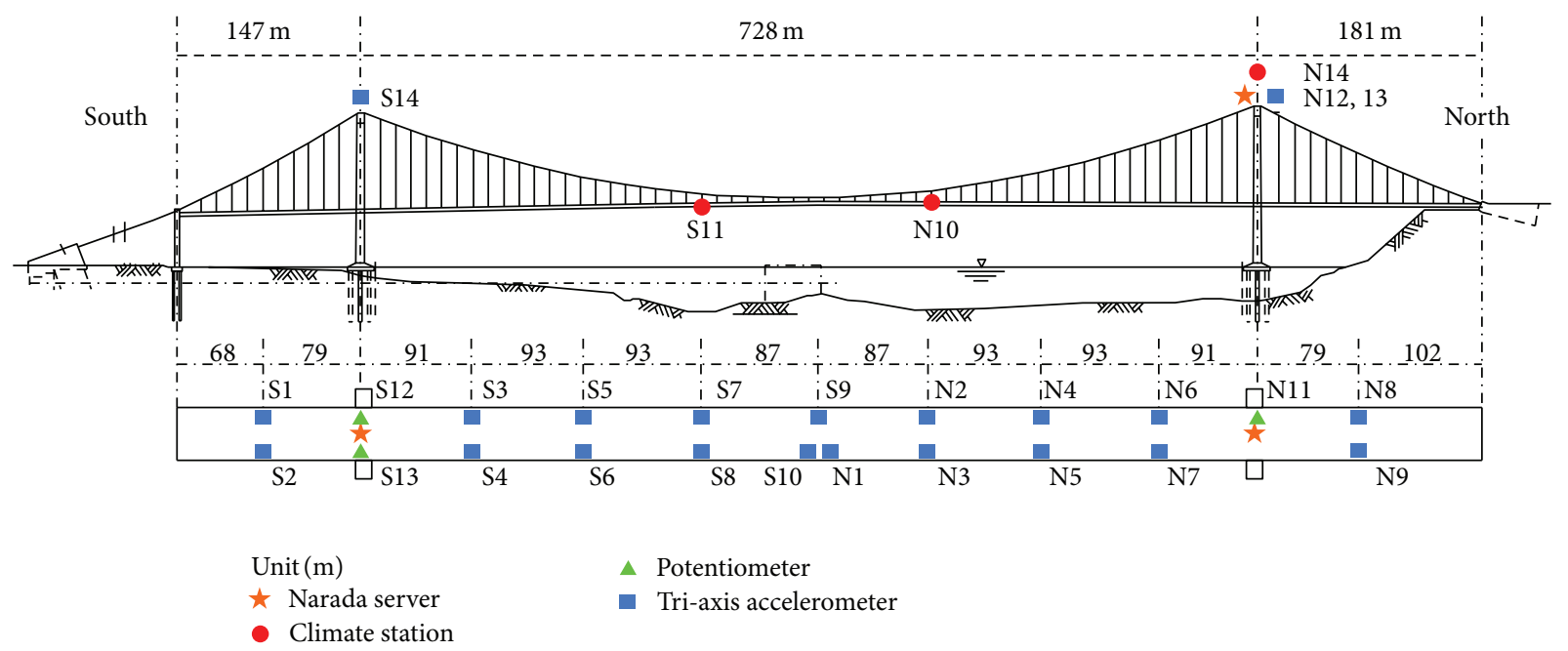

FIGURE 24: Long-term wireless monitoring system installed on the NCB: sensor types and locations [144].

the MEMS-type accelerometer was chosen to measure hanger cable tension. The electrical resistance sensor was applied onto the stiffening truss, a horizontal member, to measure strain. The propeller type wind gauge and the Thermistor type thermometer were also used. A stable power supply is secured by using a solar cell and rechargeable battery. But the solar cell is too large to be used extensively. A total of $45 \mathrm{u}$-Nodes have been installed in some of the major locations of the stiffening truss structure and in the suspension hanger cables to conduct bridge health monitoring. The USN spends about $50 \%$ of its entire system installation costs on cable installation work and data loggers. Overall, the transmission rates were found to be within a stable $90 \%$ range. Most data were lost in CDMA modems rather than in a $\mathrm{u}$-Node to $\mathrm{u}$-Gateway ZigBee communication. Although there are some data losses, the data were correctly analyzed by FFT and the tensions of hangers were calculated successfully. The test was operated successfully for about three months, which shows the wireless communication stability and sensor data reliability.

$\mathrm{Hu}$ et al. [13] deployed six wireless acceleration sensors and twenty-four strain sensors on the Zhengdian Highway Bridge in Wuhan, China. The Zhengdian Highway Bridge is a prestressed, concrete structure, simply supported slab bridge. The main bridge is $296.54 \mathrm{~m}$ long, which consists of 18 spans of $16 \mathrm{~m}$ each and two bridge abutments. The sampling rate is set as $100 \mathrm{~Hz}$. The network topology uses multihops, with up to four hops in the WSN. The test takes about an hour and a half. Packet loss was observed during the tests. The final packet delivery ratio is nearly $99.96 \%$. The mode shapes derived from six vertical acceleration measurements with the use of the multivariate ARX method correlate in terms of frequency and shape with finite element analysis. The feasibility of the proposed wireless monitoring system to capture data, transmit data via multihop wireless communication, and analyze monitoring data has been validated.

In addition, a number of other research teams have explored the performance validation and application of WSNs on real bridges. Lei et al. [147] installed a wireless structural monitoring system on the Wuyuan Bridge in China to monitor the vertical and transverse responses of the bridge riders and arch ribs. Bischoff et al. [148] deployed a WSN, which consisted of 8 nodes supplied with resistance strain gages and the root node, on critical elements to catch the real loading during the passage of heavy freight trains so as to assess the performance of the Keräsjokk Railway Bridge in Sweden. Meyer et al. [149] equipped six cable stays of the Stork Bridge with wireless accelerometers to record the cable vibration, which shows that cable stay force monitoring based on WSNs is feasible. Ye et al. [150] deployed eleven Imote2 sensor nodes on a 30-meter-long railway overpass bridge to verify the decentralized approach for the modal test. Yun and Min [97] installed 46 sensors including 16 wireless accelerometers for vibration monitoring and 5 wireless impedance sensor nodes to investigate the long-term behaviors of the Han River Railroad Bridge. Spencer Jr. and Cho [2] implemented a WSN consisting of 22 leaf nodes in the 115-year-old steel truss Government Bridge to monitor the vibration during operation.

\section{Conclusions}

WSNs with dense wireless sensors provide a potential solution for long-term, scalable SHM of bridges by providing easier installation and efficient data management at a lower cost than that of traditional tethered monitoring systems. The researchers mentioned previously have dedicated their efforts to promote the WSNs-based bridge health monitoring system with the aim of replacing wired structural monitoring systems. Many available wireless sensor systems are already quite capable and can be expected to replace the traditional wired sensor systems for bridge monitoring. However, wireless sensing technology is still in its infancy; much work remains for bringing this promising technology to fulfill the requirement of complex bridges monitoring and evaluation. The technological improvements require 
multidisciplinary researches among the engineers in civil, mechanical, electrical, and computer science engineering to facilitate the comprehensive developments for wireless SHM. Now, the main technological limitations are the power supply, data transmission reliability, and network bandwidth. Some of the existing problems and promising efforts for future research are discussed as follows.

(1) Power Supply. Each operation of the wireless sensors impacts their lifetime and consequently the lifetime of the entire WSN. Most WSN-based monitoring systems suffer from high power consumption and are generally equipped with a small power supply. Although extensive research work has been focused on energy harvest and power consumption optimization, the lifetime of a wireless sensor often falls below the levels needed for active sensing systems of bridge health monitoring. Power supply is still considered as one of the most important challenges that should be addressed for the WSNs to be more widely adopted in bridge health monitoring.

(2) Design Criteria. The hardware and software of both academic and commercial wireless monitoring systems are proposed personally, which makes extensive expertise required to master those systems. General researchers become incompetent when facing so many complicated operations, so the application of WSNs technology for monitoring bridges is severely limited. Therefore, establishing design specifications of hardware and friendly software development environment is significant so that researchers can focus on improving the performance of the WSNs-based bridge health monitoring system instead of studying elementary knowledge about WSNs.

(3) Data Management. WSNs technology enables the deployment of a dense array of sensors to monitor complicated bridges. A tremendous amount of data would be recorded every minute. But the employed low-power radio transceivers only provide a limited bandwidth. An inappropriate data management strategy may induce a large-scale WSN monitoring system collapse. On the other hand, data architecture is the key issue related to the algorithm of structural assessment which can be implemented in real time.

(4) Optimal Sensor Placement. Comparing with the needs of understanding bridge performance extensively, the sensor coverage is undoubtedly insufficient, even though the cheap wireless sensors make the installation of a lot of sensors on structures possible. Sensor placement optimization is an inevitable problem when deploying a sophisticated WSNsbased health monitoring system on a large-scale bridge. The limited radio transmission range makes the placement optimization of wireless sensors more complex than that of wired sensors.

(5) Concept. As a novel technology emerging about 15 years ago, the WSNs-based bridge health monitoring system is still an illusory thing in the concept of many administers of bridges. Although a lot of demonstrations indicate that the reliability of the WSNs is comparable with the traditional wired sensor system, the invisible data transmission path makes this technology not extensively accepted. More effort should be contributed to strengthening the convincingness of WSNs technology in the application of bridge health monitoring.

\section{Acknowledgments}

This research work was jointly supported by the Science Fund for Creative Research Groups of the NSFC (Grant no. 51121005), the National Natural Science Foundation of China (Grants nos. 51308186, 51222806, and 51278104), the Natural Science Foundation of Jiangsu Province of China (Grant no. BK20130850), and the Research Fund of the Key Laboratory of Concrete and Prestressed Concrete Structure of the Ministry of Education (Grant no. CPCSME2012-02).

\section{References}

[1] K. Wardhana and F. C. Hadipriono, "Analysis of recent bridge failures in the United States," Journal of Performance of Constructed Facilities, vol. 17, no. 3, pp. 144-150, 2003.

[2] B. F. Spencer Jr. and S. Cho, "Wireless smart sensor technology for monitoring civil infrastructure: technological developments and full-scale applications," in Proceedings of the World Congress on Advances in Structural Engineering and Mechanics (ASEM '11), Seoul, Republic of Korea, 2011.

[3] M. Bocca, E. I. Cosar, J. Salminen, and L. M. Eriksson, "A reconfigurable wireless sensor network for structural health monitoring," in Proceedings of International Society for Structural Health Monitoring of Intelligent Infrastructures (ISHMII '09), Zurich, Switzerland, 2009.

[4] M. V. Gangone, M. J. Whelan, and K. D. Janoyan, "Wireless monitoring of a multispan bridge superstructure for diagnostic load testing and system identification," Computer-Aided Civil and Infrastructure Engineering, vol. 26, no. 7, pp. 560-579, 2011.

[5] B. M. Phares, D. D. Rolander, B. A. Graybeal, and G. A. Washer, "Studying the reliability of bridge inspection," Public Roads, vol. 64, no. 3, pp. 15-19, 2000.

[6] J. M. Ko and Y. Q. Ni, “Technology developments in structural health monitoring of large-scale bridges," Engineering Structures, vol. 27, no. 12, pp. 1715-1725, 2005.

[7] T. H. Yi and H. N. Li, "Methodology developments in sensor placement for health monitoring of civil infrastructures," International Journal of Distributed Sensor Networks, vol. 2012, Article ID 612726, 11 pages, 2012.

[8] M. Çelebi, "Real-time seismic monitoring of the New Cape Girardeau Bridge and preliminary analyses of recorded data: an overview," Earthquake Spectra, vol. 22, no. 3, pp. 609-630, 2006.

[9] M. J. Chae, H. S. Yoo, J. Y. Kim, and M. Y. Cho, "Development of a wireless sensor network system for suspension bridge health monitoring," Automation in Construction, vol. 21, no. 1, pp. 237252, 2012.

[10] J. P. Lynch, A. Partridge, K. H. Law, T. W. Kenny, A. S. Kiremidjian, and E. Carryer, "Design of piezoresistive MEMSbased accelerometer for integration with wireless sensing unit for structural monitoring," Journal of Aerospace Engineering, vol. 16, no. 3, pp. 108-114, 2003.

[11] D. L. Mascarenas, M. D. Todd, G. Park, and C. R. Farrar, "Development of an impedance-based wireless sensor node for structural health monitoring," Smart Materials and Structures, vol. 16, no. 6, pp. 2137-2145, 2007. 
[12] S. Kim, S. Pakzad, D. Culler et al., "Health monitoring of civil infrastructures using wireless sensor networks," in Proceedings of the 6th International Symposium on Information Processing in Sensor Networks (IPSN '07), pp. 254-263, April 2007.

[13] X. Hu, B. Wang, and H. Ji, "A wireless sensor network-based structural health monitoring system for highway bridges," Computer-Aided Civil and Infrastructure Engineering, vol. 28, no. 3, pp. 193-209, 2013.

[14] M. J. Whelan, M. V. Gangone, and K. D. Janoyan, "Highway bridge assessment using an adaptive real-time wireless sensor network," IEEE Sensors Journal, vol. 9, no. 11, pp. 1405-1413, 2009.

[15] J. P. Lynch, K. H. Law, A. S. Kiremidjian, T. W. Kenny, E. Carryer, and A. Partridge, "The design of a wireless sensing unit for structural health monitoring," in Proceedings of the $3 \mathrm{rd}$ International Workshop on Structural Health Monitoring, pp. 1214,2001

[16] B. F. Spencer Jr., M. E. Ruiz-Sandoval, and N. Kurata, "Smart sensing technology: opportunities and challenges," Structural Control and Health Monitoring, vol. 11, no. 4, pp. 349-368, 2004.

[17] N. Kurata, B. F. Spencer Jr., and M. Ruiz-Sandoval, "Risk monitoring of buildings with wireless sensor networks," Structural Control and Health Monitoring, vol. 12, no. 3-4, pp. 315-327, 2005.

[18] E. G. Straser and A. S. Kiremidjian, "A modular, wireless damage monitoring system for structure," Report 128, John A. Blume Earthquake Center, CE Department, Stanford University, Stanford, Calif, USA, 1998.

[19] J. P. Lynch and K. J. Loh, "A summary review of wireless sensors and sensor networks for structural health monitoring," Shock and Vibration Digest, vol. 38, no. 2, pp. 91-130, 2006.

[20] M. Bocca, L. M. Eriksson, A. Mahmood, R. Jäntti, and J. Kullaa, "A synchronized wireless sensor network for experimental modal analysis in structural health monitoring," ComputerAided Civil and Infrastructure Engineering, vol. 26, no. 7, pp. 483-499, 2011.

[21] T. Nagayama, S. H. Sim, Y. Miyamori, and B. F. Spencer Jr., "Issues in structural health monitoring employing smart sensors," Smart Structures and Systems, vol. 3, no. 3, pp. 299329, 2007.

[22] V. Krishnamurthy, K. Fowler, and E. Sazonov, "The effect of time synchronization of wireless sensors on the modal analysis of structures," Smart Materials and Structures, vol. 17, no. 5, Article ID 055018, 2008.

[23] S. Cho, C. B. Yun, J. P. Lynch, A. T. Zimmerman, B. F. Spencer Jr., and T. Nagayama, "Smart wireless sensor technology for structural health monitoring of civil structures," International Journal of Steel Structures, vol. 8, no. 4, pp. 267-275, 2008.

[24] T. Nagayama, B. F. Spencer Jr., and J. A. Rice, "Structural health monitoring utilizing Intel's Imote2 wireless sensor platform," in Proceedings of the Sensors and Smart Structures Technologies for Civil, Mechanical, and Aerospace Systems, Yokohama, Japan, March 2007.

[25] H. Jo, J. A. Rice, B. F. Spencer Jr., and T. Nagayama, "Development of high-sensitivity accelerometer board for structural health monitoring," in Sensors and Smart Structures Technologies for Civil, Mechanical, and Aerospace Systems, vol. 7647 of Proceedings of SPIE, March 2010.

[26] M. Ruiz-Sandoval, T. Nagayama, and B. F. Spencer Jr., "Sensor development using Berkeley Mote platform," Journal of Earthquake Engineering, vol. 10, no. 2, pp. 289-309, 2006.
[27] J. P. Lynch, K. H. Law, A. S. Kiremidjian, T. Kenny, and E. Carryer, "A wireless modular monitoring system for civil structures," in Proceedings of the International Modal Analysis Conference (IMAC '02), February 2002.

[28] J. P. Lynch, K. H. Law, A. S. Kiremidjian et al., "Validation of a wireless modular monitoring system for structures," in SPIE's 9th Annual International Symposium on Smart Structures and Materials, Proceedings of SPIE, pp. 124-135, March 2002.

[29] J. P. Lynch, A. Sundararajan, K. H. Law et al., "Field validation of a wireless structural monitoring system on the Alamosa Canyon Bridge," in Smart Structures and Materials. Smart Systems and Nondestructive Evaluation for Civil Infrastructures, Proceedings of SPIE, pp. 267-278, March 2003.

[30] J. P. Lynch, A. Sundararajan, and K. H. Law, "Design and performance validation of a wireless sensing unit for structural health monitoring applications," Structural Engineering and Mechanics, no. 173, pp. 393-408, 2004.

[31] J. P. Lynch, G. Parra-Montesinos, B. A. Canbolat, and T. C. Hou, "Real-time damage prognosis of high-performance fiber reinforced cementitious composite structures," in Proceeding of Advances in Structural Engineering and Mechanics (ASEM '04), Seoul, Republic of Korea, 2004.

[32] Y. Wang, J. P. Lynch, and K. H. Law, "Wireless structural sensors using reliable communication protocols for data acquisition and interrogation," in Proceedings of the 23rd International Modal Analysis Conference (IMAC '05), 2005.

[33] Y. Wang, J. P. Lynch, and K. H. Law, "Design of a lowpower wireless structural monitoring system for collaborative computational algorithms," in Nondestructive Evaluation for Health Monitoring and Diagnostics, Proceedings of SPIE, pp. 106-117, March 2005.

[34] Y. Wang, J. P. Lynch, and K. H. Law, “A wireless structural health monitoring system with multithreaded sensing devices: design and validation," Structure and Infrastructure Engineering, vol. 3, no. 2, pp. 103-120, 2007.

[35] R. A. Swartz, D. Jung, J. P. Lynch, Y. Wang, D. Shi, and M. Flynn, "Design of a wireless sensor for scalable distributed innetwork computation in a structural health monitoring system," in Proceedings of the 5th International Workshop on Structural Health Monitoring, pp. 1570-1577, 2005.

[36] A. T. Zimmerman, M. Shiraishi, R. A. Swartz, and J. P. Lynch, "Automated modal parameter estimation by parallel processing within wireless monitoring systems," Journal of Infrastructure Systems, vol. 14, no. 1, pp. 102-113, 2008.

[37] A. T. Zimmerman and J. P. Lynch, "A parallel simulated annealing architecture for model updating in wireless sensor networks," IEEE Sensors Journal, vol. 9, no. 11, pp. 1503-1510, 2009.

[38] G. Heo, W. Lee, G. Lee, and D. Lee, "Identifying dynamic characteristics of structures to estimate the performance of a smart wireless MA system," in Smart Structures and Materials. Smart Sensor Technology and Measurement Systems, Proceedings of SPIE, pp. 373-380, March 2005.

[39] G. Heo, W. Lee, G. Lee, J.-R. Jeon, and C. Kim, "Field validation of a wireless sensor unit using self-anchored suspension bridge," in Smart Structures and Materials, Proceedings of SPIE, March 2006.

[40] C. Park, Q. Xie, P. H. Chou, and M. Shinozuka, "DuraNode: wireless networked sensor for structural health monitoring," in Proceedings of the 4th IEEE Conference on Sensors, pp. 277-280, November 2005. 
[41] H. Joshi, B. Burlacu, M. Prabhu et al., "Wireless structural health monitoring system design, implementation and validation," Research Report 06-02, Constructed Facilities Lab (CFL), North Carolina State University (NCSU), Raleigh, NC, USA, 2006.

[42] S. N. Pakzad, G. L. Fenves, S. Kim, and D. E. Culler, "Design and implementation of scalable wireless sensor network for structural monitoring," Journal of Infrastructure Systems, vol. 14, no. 1, pp. 89-101, 2008.

[43] J.-H. Park, J.-T. Kim, D.-S. Hong, D. Mascarenas, and J. Peter Lynch, "Autonomous smart sensor nodes for global and local damage detection of prestressed concrete bridges based on accelerations and impedance measurements," Smart Structures and Systems, vol. 6, no. 5-6, pp. 711-730, 2010.

[44] J. A. Rice and B. F. Spencer Jr., "Structural health monitoring sensor development for the Imote2 platform," in Sensors and Smart Structures Technologies for Civil, Mechanical, and Aerospace Systems, vol. 6932 of Proceedings of SPIE, March 2008.

[45] N. Kurata, S. Saruwatari, and H. Morikawa, "Ubiquitous structural monitoring using wireless sensor networks," in Proceedings of the International Symposium on Intelligent Signal Processing and Communications (ISPACS '06), pp. 99-102, December 2006.

[46] H. Jo, S. H. Sim, T. Nagayama, and B. F. Spencer Jr., "Development and application of high-sensitivity wireless smart sensors for decentralized stochastic modal identification," Journal of Engineering Mechanics, vol. 138, no. 6, pp. 683-694, 2012.

[47] H. Jo, S.-H. Sim, K. A. Mechitov et al., "Hybrid wireless smart sensor network for full-scale structural health monitoring of a cable-stayed bridge," in Sensors and Smart Structures Technologies for Civil, Mechanical, and Aerospace Systems, Proceedings of SPIE, March 2011.

[48] M. J. Whelan and K. D. Janoyan, "Design of a robust, high-rate wireless sensor network for static and dynamic structural monitoring," Journal of Intelligent Material Systems and Structures, vol. 20, no. 7, pp. 849-863, 2009.

[49] B. Chen and W. Liu, "Mobile agent computing paradigm for building a flexible structural health monitoring sensor network," Computer-Aided Civil and Infrastructure Engineering, vol. 25, no. 7, pp. 504-516, 2010.

[50] M. L. Wang, H. Gu, G. M. Lloyd, and Y. Zhaok, "A multichannel wireless PVDF displacement sensor for structural monitoring," in Proceedings of the International Workshop on Advanced Sensors, Structural Health Monitoring and Smart Structures, Tokyo, Japan, 2003.

[51] K. J. Loh, J. P. Lynch, and N. A. Kotov, "Passive wireless sensing using SWNT-based multifunctional thin film patches," International Journal of Applied Electromagnetics and Mechanics, vol. 28, no. 1-2, pp. 87-94, 2008.

[52] T. Harms, B. Banks, S. S. Sarvestani, and F. Bastianini, "Design and testing of a low-power wireless sensor network for structural health monitoring of bridges," in Sensors and Smart Structures Technologies for Civil, Mechanical, and Aerospace Systems, Proceedings of SPIE, 72920U-8, March 2009.

[53] C. O. Yang, M. Yen, and N. Weng, "The development of an on-line structural health monitoring system based on wireless sensor networks," Electronic Journal of Structural Engineering, 2010.

[54] S. G. Taylor, K. M. Farinholt, G. Park, M. D. Todd, and C. R. Farrar, "Multi-scale wireless sensor node for health monitoring of civil infrastructure and mechanical systems," Smart Structures and Systems, vol. 6, no. 5-6, pp. 661-673, 2010.
[55] J. Min, S. Park, C.-B. Yun, and B. Song, "Development of a lowcost multifunctional wireless impedance sensor node," Smart Structures and Systems, vol. 6, no. 5-6, pp. 689-709, 2010.

[56] S. Zakikhani, A. Bagchi, and A. Hammad, "Design criteria of wireless sensors in structural health monitoring systems," in Proceedings of the International Conference on Computing in Civil and Building Engineering, vol. 30, p. 445, 2010.

[57] J. P. Lynch, K. H. Law, A. S. Kiremidjian, T. W. Kenny, E. Carryer, and A. Partridge, "The design of a wireless sensing unit for structural health monitoring," in Proceedings of the 3 rd International Workshop on Structural Health Monitoring, pp. 1214, 2001.

[58] Y. Gao, B. F. Spencer Jr., and M. Ruiz-Sandoval, "Distributed computing strategy for structural health monitoring," Structural Control and Health Monitoring, vol. 13, no. 1, pp. 488-507, 2006.

[59] J. Lian, K. Naik, and G. B. Agnew, "Data capacity improvement of wireless sensor networks using non-uniform sensor distribution," International Journal of Distributed Sensor Networks, vol. 2, no. 2, pp. 121-145, 2006.

[60] J. A. Rice, K. Mechitov, S.-H. Sim et al., "Flexible smart sensor framework for autonomous structural health monitoring," Smart Structures and Systems, vol. 6, no. 5-6, pp. 423-438, 2010.

[61] J. P. Lynch, Decentralization of wireless monitoring and control technologies for smart civil structures [Ph.D. thesis], Department of Civil and Environmental Engineering, Stanford University, Stanford, Calif, USA, 2002.

[62] J. P. Lynch, A. Sundararajan, K. H. Law, A. S. Kiremidjian, T. Kenny, and E. Carryer, "Embedment of structural monitoring algorithms in a wireless sensing unit," Structural Engineering and Mechanics, vol. 15, no. 3, pp. 285-297, 2003.

[63] J. P. Lynch, A. Sundararajan, K. H. Law, A. S. Kiremidjian, and E. Carryer, "Power-efficient data management for a wireless structural monitoring system," in Proceedings of the 4th International Workshop on Structural Health Monitoring, vol. 1, pp. 15-17, Stanford University, November 2003.

[64] J. P. Lynch, A. Sundararajan, K. H. Law, A. S. Kiremidjian, and E. Carryer, "Embedding damage detection algorithms in a wireless sensing unit for operational power efficiency," Smart Materials and Structures, vol. 13, no. 4, pp. 800-810, 2004.

[65] A. S. Kiremidjian, G. Kiremidjian, and P. Sarabandi, "A wireless structural monitoring system with embedded damage algorithms and decision support system," Structure and Infrastructure Engineering, vol. 7, no. 12, pp. 881-894, 2011.

[66] J. Caffrey, R. Govindan, and E. Johnson, "Networked sensing for structural health monitoring," in Proceedings of the 4th International Workshop on Structural Control, pp. 57-66, Columbia University, 2004.

[67] Y. Hashimoto, A. Masuda, and A. Sone, "Prototype of sensor network with embedded local data processing," in Smart Structures and Materials 2005. Sensors and Smart Structures Technologies for Civil, Mechanical, and Aerospace Systems, Proceedings of SPIE, pp. 245-252, March 2005.

[68] K. Chintalapudi, E. A. Johnson, and R. Govindan, "Structural damage detection using wireless sensor-actuator networks," in Proceedings of the IEEE International Symposium on, Mediterrean Conference on Control and Automation, pp. 322-327, June 2005.

[69] S. Cho, J. P. Lynch, and C.-B. Yun, "Development of a low-cost automated tension estimation system for cable-stayed bridges," in Proceedings of the ASME Conference on Smart Materials, Adaptive Structures and Intelligent Systems (SMASIS '08), pp. 279-287, October 2008. 
[70] S. Cho, J. P. Lynch, J.-J. Lee, and C.-B. Yun, "Development of an automated wireless tension force estimation system for cable-stayed bridges," Journal of Intelligent Material Systems and Structures, vol. 21, no. 3, pp. 361-376, 2010.

[71] H. Zui, T. Shinke, and Y. Namita, "Practical formulas for estimation of cable tension by vibration method," Journal of Structural Engineering, vol. 122, no. 6, pp. 651-656, 1996.

[72] N. Castaneda, F. Sun, S. Dyke, C. Lu, A. Hope, and T. Nagayama, "Implementation of a correlation-based decentralized damage detection method using wireless sensors," in Proceedings of the ASEM Conference, 2008.

[73] G. Hackmann, F. Sun, N. Castaneda, C. Lu, and S. Dyke, "A holistic approach to decentralized structural damage localization using wireless sensor networks," in Proceedings of the RealTime Systems Symposium (RTSS '08), pp. 35-46, December 2008.

[74] S. H. Sim and B. F. Spencer Jr., "Decentralized strategies for monitoring structures using wireless smart sensor networks," NSEL Report Series, no. 19, 2009.

[75] S. H. Sim, J. Li, S. Cho et al., "Automated cable tension monitoring using smart sensors," in Proceedings of the World Congress on Advances in Structural Engineering and Mechanics (ASEM '11), 2011.

[76] T. Shimada, "Estimating method of cable tension from natural frequency of high mode," Proceedings of the Japan Society of Civil Engineers, no. 501, pp. 163-171, 1994.

[77] T.-Y. Hsu, S.-K. Huang, K.-C. Lu, C.-H. Loh, Y. Wang, and J. P. Lynch, "On-line structural damage localization and quantification using wireless sensors," Smart Materials and Structures, vol. 20, no. 10, Article ID 105025, 2011.

[78] J. Kim and J. P. Lynch, "Autonomous decentralized system identification by Markov parameter estimation using distributed smart wireless sensor networks," Journal of Engineering Mechanics, vol. 138, no. 5, pp. 478-490, 2011.

[79] G. J. Yun, S.-G. Lee, J. Carletta, and T. Nagayama, “Decentralized damage identification using wavelet signal analysis embedded on wireless smart sensors," Engineering Structures, vol. 33, no. 7, pp. 2162-2172, 2011.

[80] J. Zhang, "On-board computing for structural health monitoring with smart wireless sensors by modal identification using Hilbert-Huang transform," Mathematical Problems in Engineering, vol. 2013, Article ID 509129, 9 pages, 2013.

[81] S.-H. Sim, J. F. Carbonell-Mrquez, B. F. Spencer Jr., and H. Jo, "Decentralized random decrement technique for efficient data aggregation and system identification in wireless smart sensor networks," Probabilistic Engineering Mechanics, vol. 26, no. 1, pp. 81-91, 2011.

[82] Y. Gao and B. F. Spencer Jr., "Online damage diagnosis for civil infrastructure employing a flexibility-based approach," Smart Materials and Structures, vol. 15, no. 1, pp. 9-19, 2006.

[83] D. Bernal, "Load vectors for damage localization," Journal of Engineering Mechanics, vol. 128, no. 1, pp. 7-14, 2002.

[84] Y. Gao and B. F. Spencer Jr., "Structural health monitoring strategies for smart sensor networks," University of Illinois at Urbana-Champaign, 2005.

[85] T. Nagayama and B. F. Spencer Jr., "Structural health monitoring using smart sensors," Newmark Structural Engineering Laboratory (NSEL) Report Series 1, University of Illinois at UrbanaChampaign, Urbana, Ill, USA, 2007.

[86] S. H. Sim, B. F. Spencer Jr., M. Zhang, and H. Xie, "Automated decentralized modal analysis using smart sensors," Structural Control and Health Monitoring, vol. 17, no. 8, pp. 872-894, 2010.
[87] D. Bernal, "Flexibility-based damage localization from stochastic realization results," Journal of Engineering Mechanics, vol. 132, no. 6, pp. 651-658, 2006.

[88] G. Yan, W. Guo, S. J. Dyke, G. Hackmann, and C. Lu, "Experimental validation of a multi-level damage localization technique with distributed computation," Smart Structures and Systems, vol. 6, no. 5-6, pp. 561-578, 2010.

[89] G. Hackmann, W. Guo, G. Yan, C. Lu, and S. Dyke, "Cyberphysical codesign of distributed structural health monitoring with wireless sensor networks," in Proceedings of the 1st ACM/IEEE International Conference on Cyber-Physical Systems (ICCPS '10), pp. 119-128, April 2010.

[90] J.-T. Kim, J.-H. Park, D.-S. Hong, and W.-S. Park, "Hybrid health monitoring of prestressed concrete girder bridges by sequential vibration-impedance approaches," Engineering Structures, vol. 32, no. 1, pp. 115-128, 2010.

[91] A. T. Zimmerman and J. P. Lynch, "Market-based frequency domain decomposition for automated mode shape estimation in wireless sensor networks," Structural Control and Health Monitoring, vol. 17, no. 7, pp. 808-824, 2010.

[92] E. Sazonov, K. Janoyan, and R. Jha, "Wireless intelligent sensor network for autonomous structural health monitoring," in Smart Structures and Materials. Smart Sensor Technology and Measurement Systems, Proceedings of SPIE, pp. 305-314, usa, March 2004.

[93] J. H. Galbreath, C. P. Townsend, and S. W. Mundell, "Civil structure strain monitoring with power-efficient high-speed wireless sensor networks," in Proceedings International Workshop for Structural Health Monitoring, vol. 3, Stanford, Calif, USA, 2003.

[94] A. T. Zimmerman, J. P. Lynch, and F. T. Ferrese, "Market-based resource allocation for distributed data processing in wireless sensor networks," ACM Transactions on Embedded Computing Systems, vol. 12, no. 3, article 84, 2013.

[95] T. S. Fu, A. Ghosh, E. A. Johnson, and B. Krishnamachari, "Energy-efficient deployment strategies in structural health monitoring using wireless sensor networks," Structural Control and Health Monitoring, vol. 20, pp. 971-986, 2013.

[96] H. A. Sodano, D. J. Inman, and G. Park, "A review of power harvesting from vibration using piezoelectric materials," Shock and Vibration Digest, vol. 36, no. 3, pp. 197-205, 2004.

[97] C. B. Yun and J. Min, "Smart sensing, monitoring, and damage detection for civil infrastructures," KSCE Journal of Civil Engineering, vol. 15, no. 1, pp. 1-14, 2011.

[98] D. D. L. Mascarenas, E. Flynn, K. Lin et al., "Demonstration of a roving-host wireless sensor network for rapid assessment monitoring of structural health," in Smart Sensor Phenomena, Technology, Networks, and Systems, Proceedings of SPIE, 69330K-1, March 2008.

[99] H.-J. Park, H. Sohn, C.-B. Yun, J. Chung, and I. I.-B. Kwon, "A wireless guided wave excitation technique based on laser and optoelectronics," Smart Structures and Systems, vol. 6, no. 5-6, pp. 749-765, 2010.

[100] P. Glynne-Jones and N. M. White, "Self-powered systems: a review of energy sources," Sensor Review, vol. 21, no. 2, pp. 91-97, 2001.

[101] S. Roundy, P. K. Wright, and J. M. Rabaey, Energy Scavenging for Wireless Sensor Networks: With Special Focus on Vibrations, Springer, 2004.

[102] M. A. Qidwai, J. P. Thomas, J. C. Kellogg, and J. Baucom, "Energy harvesting concepts for small electric unmanned systems," in Smart Structures and Materials. Active Materials: 
Behaviour and Mechanics, Proceedings of SPIE, pp. 84-85, March 2004.

[103] L. Mateu and F. Moll, "Review of energy harvesting techniques and applications for microelectronics," in VLSI Circuits and Systems II, vol. 5837 of Proceedings of SPIE, pp. 359-373, May 2005.

[104] J. A. Paradiso and T. Starner, "Energy scavenging for mobile and wireless electronics," IEEE Pervasive Computing, vol. 4, no. 1, pp. 18-27, 2005.

[105] V. Raghunathan, A. Kansal, J. Hsu, J. Friedman, and M. Srivastava, "Design considerations for solar energy harvesting wireless embedded systems," in Proceedings of the 4th International Symposium on Information Processing in Sensor Networks (IPSN '05), vol. 64, pp. 457-462, IEEE Press, April 2005.

[106] S. Jang, H. Jo, S. Cho et al., "Structural health monitoring of a cable-stayed bridge using smart sensor technology: deployment and evaluation," Smart Structures and Systems, vol. 6, no. 5-6, pp. 439-459, 2010.

[107] R. Anant Kishore and S. Priya, "Design and experimental verification of a high efficiency small wind energy portable turbine (SWEPT)," Journal of Wind Engineering and Industrial Aerodynamics, vol. 118, pp. 12-19, 2013.

[108] W. K. G. Seah, A. E. Zhi, and H.-P. Tan, "Wireless sensor networks powered by ambient energy harvesting (WSN-HEAP) survey and challenges," in Proceedings of the 1st International IEEE Conference on Wireless Communication, Vehicular Technology, Information Theory and Aerospace and Electronic Systems Technology, Wireless (VITAE '09), pp. 1-5, May 2009.

[109] N. E. Dutoit, B. L. Wardle, and S. G. Kim, "Design considerations for MEMS-scale piezoelectric mechanical vibration energy harvesters," Integrated Ferroelectrics, vol. 71, no. 1, pp. 121-160, 2005.

[110] G. Park, T. Rosing, M. D. Todd, C. R. Farrar, and W. Hodgkiss, "Energy harvesting for structural health monitoring sensor networks," Journal of Infrastructure Systems, vol. 14, no. 1, pp. 64-79, 2008.

[111] J. M. Gilbert and F. Balouchi, "Comparison of energy harvesting systems for wireless sensor networks," International Journal of Automation and Computing, vol. 5, no. 4, pp. 334-347, 2008.

[112] A. Khaligh, P. Zeng, and C. Zheng, "Kinetic energy harvesting using piezoelectric and electromagnetic technologiesstate of the art," IEEE Transactions on Industrial Electronics, vol. 57, no. 3 , pp. 850-860, 2010.

[113] D. G. Kim, S. N. Yun, Y. B. Ham, and J. H. Park, "Energy harvesting strategy using piezoelectric element driven by vibration method," Wireless Sensor Network, vol. 2, no. 2, pp. 100-107, 2010.

[114] F. Sivrikaya and B. Yener, "Time synchronization in sensor networks: a survey," IEEE Network, vol. 18, no. 4, pp. 45-50, 2004.

[115] I.-K. Rhee, J. Lee, J. Kim, E. Serpedin, and Y.-C. Wu, “Clock synchronization in wireless sensor networks: an overview," Sensors, vol. 9, no. 1, pp. 56-85, 2009.

[116] S. M. Lasassmeh and J. M. Conrad, "Time synchronization in wireless sensor networks: a survey," in Proceedings of the IEEE SoutheastCon Conference: Energizing our Future (SoutheastCon '10), pp. 242-245, March 2010.

[117] P. Ranganathan and K. Nygard, "Time synchronization in wireless sensor networks: a survey," International Journal of UbiComp, vol. 1, no. 2, pp. 92-102, 2010.

[118] Y. Yang, Time Synchronization in Wireless Sensor Networks: A Survey, Mid Sweden University, 2012.
[119] M. Maróti, B. Kusy, G. Simon, and Á. Lédeczi, “The flooding time synchronization protocol," in Proceedings of the 2nd International Conference on Embedded Networked Sensor Systems (SenSys '04), pp. 39-49, ACM, November 2004.

[120] S. Ganeriwal, R. Kumar, and M. B. Srivastava, "Timing-sync protocol for sensor networks," in Proceedings of the Ind International Conference on Embedded Networked Sensor Systems (SenSys '03), pp. 138-149, ACM, November 2003.

[121] M. J. Whelan, M. V. Gangone, K. D. Janoyan, and R. Jha, "Wireless vibration monitoring for damage detection of highway bridges," in Smart Sensor Phenomena, Technology, Networks, and Systems, Proceedings of SPIE, 69330J-1, March 2008.

[122] M. J. Whelan, M. P. Fuchs, M. V. Gangone, and K. D. Janoyan, "Development of a wireless bridge monitoring system for condition assessment using hybrid techniques," in Sensor Systems and Networks: Phenomena, Technology, and Applications for NDE and Health Monitoring, vol. 6530 of Proceedings of SPIE, 65300H-1, March 2007.

[123] M. J. Whelan, M. V. Gangone, K. D. Janoyan, K. Cross, and R. Jha, "Reliable high-rate bridge monitoring using dense wireless sensor arrays," in Proceedings of the International Workshop on Structural Health Monitoring, 2007.

[124] S. Aoki, Y. Fujino, and M. Abe, "Intelligent bridge maintenance system using MEMS and network technology," in Smart Structures and Materials 2003: Smart Systems and Nondestructive Evaluation for Civil Infrastructures, Proceedings of SPIE, pp. 37-42, Virginia Polytechnic Institute and State University, San Diego, Calif, USA, March 2003.

[125] J. Binns, “Technology: bridge sensor system delivers results quickly," Civil Engineering-ASCE, vol. 74, no. 9, pp. 30-31, 2004.

[126] H.-C. Chung, T. Enotomo, K. Loh, and M. Shinozuka, "Realtime visualization of bridge structural response through wireless MEMS sensors," in Testing, Reliability, and Application of Micro and Nano-Material Systems II, Proceedings of SPIE, pp. 239-246, March 2004.

[127] J. P. Lynch, Y. Wang, K. H. Law, J. H. Yi, C. G. Lee, and C. B. Yun, "Validation of a large-scale wireless structural monitoring system on the Geumdang Bridge," in Proceedings of 9th International Conference on Structural Safety and Reliability, pp. 19-23, 2005.

[128] J. P. Lynch, Y. Wang, K. J. Loh, J.-H. Yi, and C.-B. Yun, "Performance monitoring of the Geumdang Bridge using a dense network of high-resolution wireless sensors," Smart Materials and Structures, vol. 15, no. 6, article 008, pp. 1561-1575, 2006.

[129] S. N. Pakzad, "Development and deployment of large scale wireless sensor network on a long-span bridge," Smart Structures and Systems, vol. 6, no. 5-6, pp. 525-543, 2010.

[130] S. N. Pakzad and G. L. Fenves, "Statistical analysis of vibration modes of a suspension bridge using spatially dense wireless sensor network," Journal of Structural Engineering, vol. 135, no. 7, pp. 863-872, 2009.

[131] J.-H. Weng, C.-H. Loh, J. P. Lynch, K.-C. Lu, P.-Y. Lin, and Y. Wang, "Output-only modal identification of a cable-stayed bridge using wireless monitoring systems," Engineering Structures, vol. 30, no. 7, pp. 1820-1830, 2008.

[132] M. J. Whelan, M. V. Gangone, K. D. Janoyan, and R. Jha, "Real-time wireless vibration monitoring for operational modal analysis of an integral abutment highway bridge," Engineering Structures, vol. 31, no. 10, pp. 2224-2235, 2009.

[133] M. V. Gangone, M. J. Whelan, K. D. Janoyan, and R. Jha, "Field deployment of a dense wireless sensor network for condition 
assessment of a bridge superstructure," in Smart Sensor Phenomena, Technology, Networks, and Systems, Proceedings of SPIE, 69330L-9, March 2008.

[134] M. J. Whelan, M. V. Gangone, K. D. Janoyan, N. A. Hoult, C. R. Middleton, and K. Soga, "Wireless operational modal analysis of a multi-span prestressed concrete bridge for structural identification," Smart Structures and Systems, vol. 6, no. 5-6, pp. 579-593, 2010.

[135] M. J. Whelan, M. V. Gangone, K. D. Janoyan, and R. Jha, "Operational modal analysis of a multi-span skew bridge using real-time wireless sensor networks," JVC/Journal of Vibration and Control, vol. 17, no. 13, pp. 1952-1963, 2011.

[136] M. J. Whelan and K. D. Janoyan, "In-service diagnostics of a highway bridge from a progressive damage case study," Journal of Bridge Engineering, vol. 15, no. 5, pp. 597-607, 2010.

[137] S. Cho, H. Jo, S. Jang et al., "Structural health monitoring of a cable-stayed bridge using wireless smart sensor technology: data analyses," Smart Structures and Systems, vol. 6, no. 5-6, pp. 461-480, 2010.

[138] J. Kim, R. A. Swartz, J. P. Lynch, J.-J. Lee, and C.-G. Lee, “Rapidto-deploy reconfigurable wireless structural monitoring systems using extended-range wireless sensors," Smart Structures and Systems, vol. 6, no. 5-6, pp. 505-524, 2010.

[139] J. Kim, J. P. Lynch, D. Zonta, J.-J. Lee, and C.-B. Yun, "Reconfigurable wireless monitoring systems for bridges: validation on the Yeondae Bridge," in Sensors and Smart Structures Technologies for Civil, Mechanical, and Aerospace Systems, Proceedings of SPIE, March 2009.

[140] N. A. Hoult, P. R. A. Fidler, P. G. Hill, and C. R. Middleton, "Long-term wireless structural health monitoring of the ferriby road bridge," Journal of Bridge Engineering, vol. 15, no. 2, pp. 153 $159,2010$.

[141] F. Stajano, N. Hoult, I. Wassell, P. Bennett, C. Middleton, and K. Soga, "Smart bridges, smart tunnels: transforming wireless sensor networks from research prototypes into robust engineering infrastructure," Ad Hoc Networks, vol. 8, no. 8, pp. 872-888, 2010.

[142] M. Kurata, J. P. Lynch, G. W. Van Der Linden, P. Hipley, and L.-H. Sheng, "Application of an automated wireless structural monitoring system for long-span suspension bridges," in Proceedings of the AIP Conference, vol. 1335, pp. 33-40, July 2010.

[143] M. Kurata, J. Kim, Y. Zhang et al., "Long-term assessment of an autonomous wireless structural health monitoring system at the new Carquinez Suspension Bridge," in Smart Structures and Materials Nondestructive Evaluation and Health Monitoring, Proceedings of SPIE, 798312-9, March 2011.

[144] M. Kurata, J. Kim, J. P. Lynch et al., "Internet-enabled wireless structural monitoring systems: development and permanent deployment at the new carquinez suspension bridge," Journal of Structural Engineering, vol. 39, no. 10, pp. 1688-1702, 2013.

[145] M. Kurata, J. P. Lynch, G. W. van der Linden, V. Jacob, and P. Hipley, "Preliminary study of a wireless structural monitoring system for the new carquinez suspension bridge," in Proceedings of the 5th World Conference on Structural Control and Monitoring, 2010.

[146] J. Kim and J. P. Lynch, "Experimental analysis of vehiclebridge interaction using a wireless monitoring system and a two-stage system identification technique," Mechanical Systems and Signal Processing, vol. 28, pp. 3-19, 2012.

[147] Y. Lei, W. A. Shen, Y. Song, and J. P. Lynch, "Application of wireless monitoring system for the ambient vibration study of the Wuyuan steel arch bridge," in Proceedings of the World Forum on Smart Materials and Smart Structures Technology (SMSST '07), pp. 149-150, Nanjing, China, May 2007.

[148] R. Bischoff, J. Meyer, O. Enochsson, G. Feltrin, and L. Elfgren, "Event-based strain monitoring on a railway bridge with a wireless sensor network," in Proceedings of 4th International Conference on Structural Health Monitoring of Intelligent Infrastructure, 2009.

[149] J. Meyer, R. Bischoff, G. Feltrin, and M. Motavalli, "Wireless sensor networks for long-term structural health monitoring," Smart Structures and Systems, vol. 6, no. 3, pp. 263-275, 2010.

[150] X. Ye, T. Zhu, Q. Yan, and W. Wang, "Experimental verification of decentralized approach for modal identification based on wireless smart sensor network," Advanced Materials Research, vol. 291-294, pp. 3-11, 2011. 


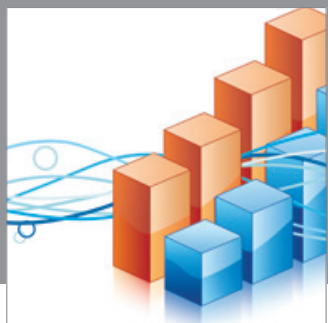

Advances in

Operations Research

mansans

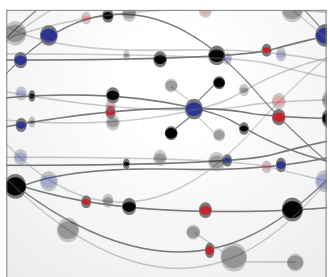

The Scientific World Journal
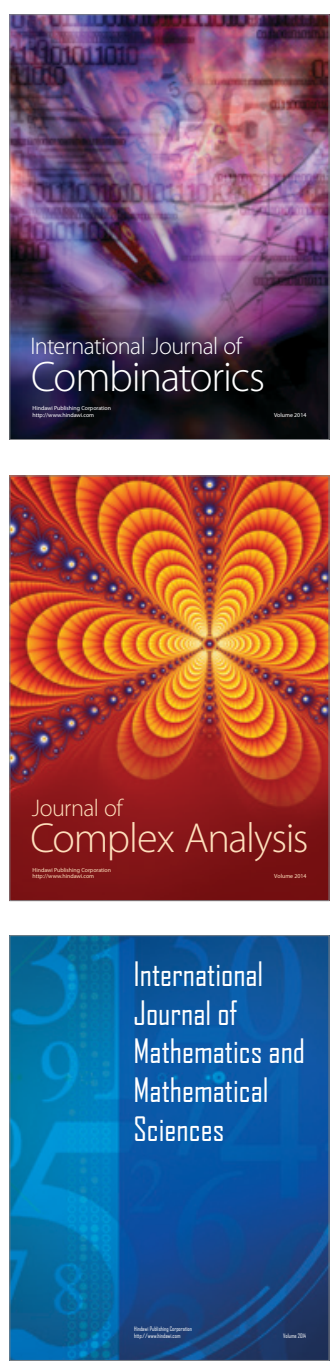
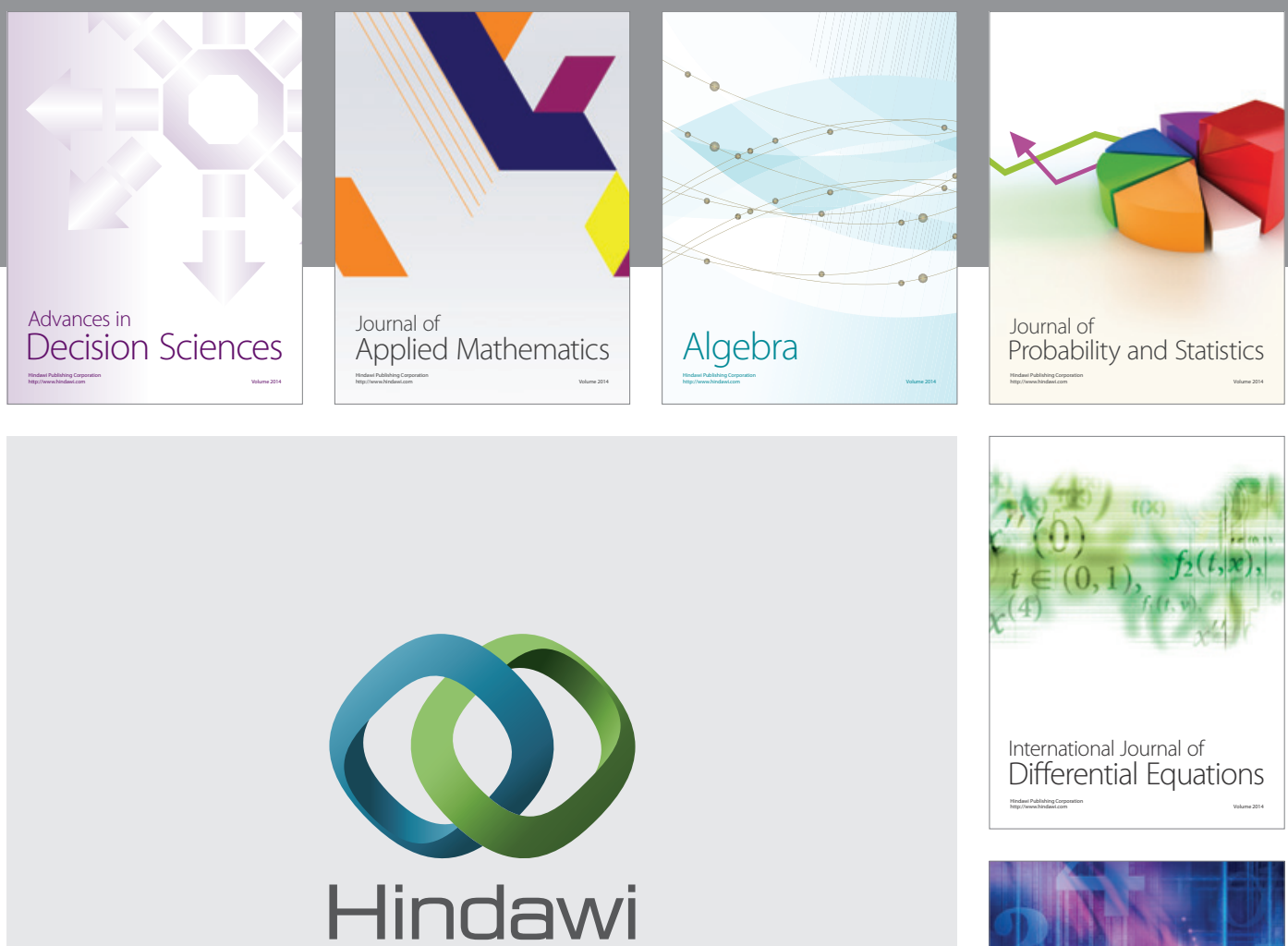

Submit your manuscripts at http://www.hindawi.com
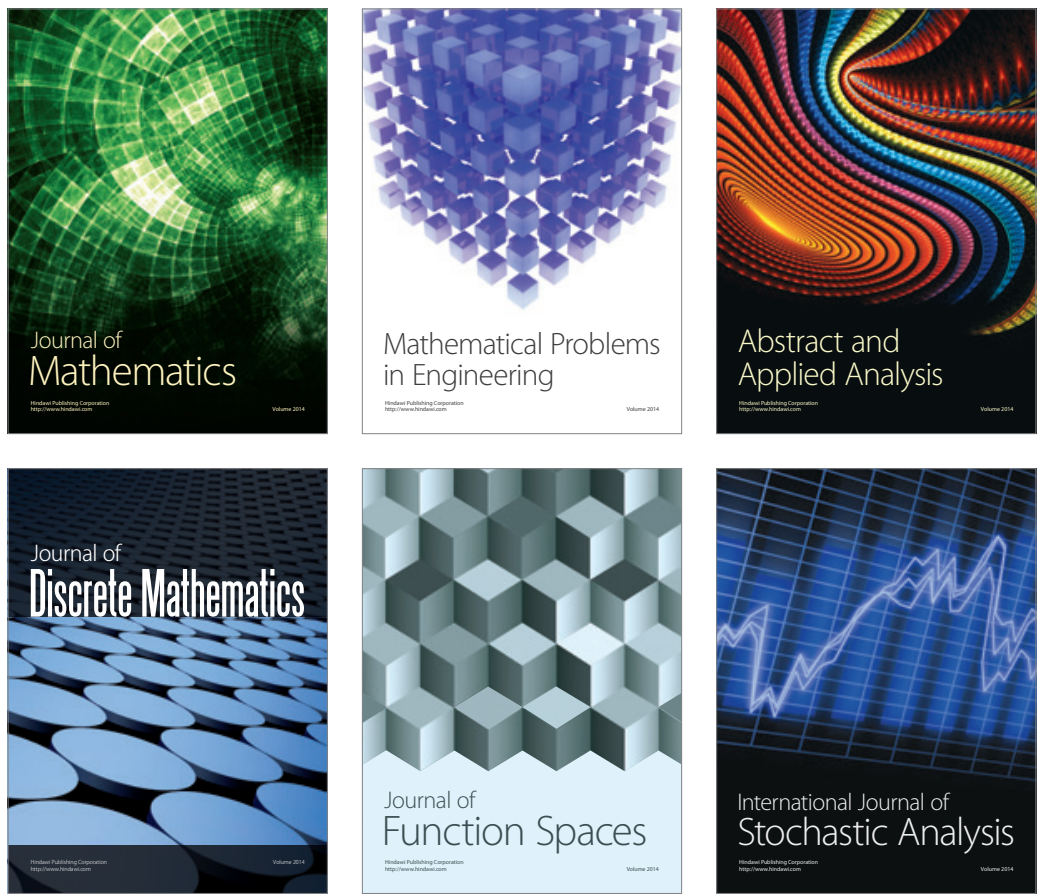

Journal of

Function Spaces

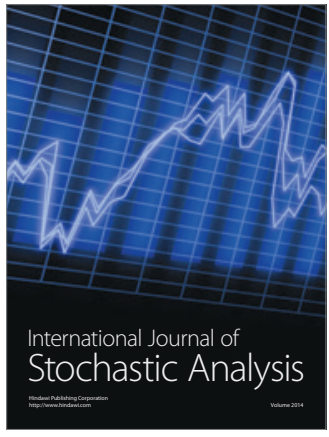

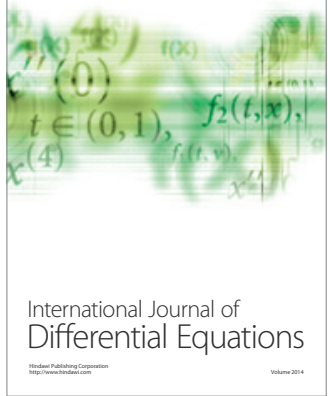
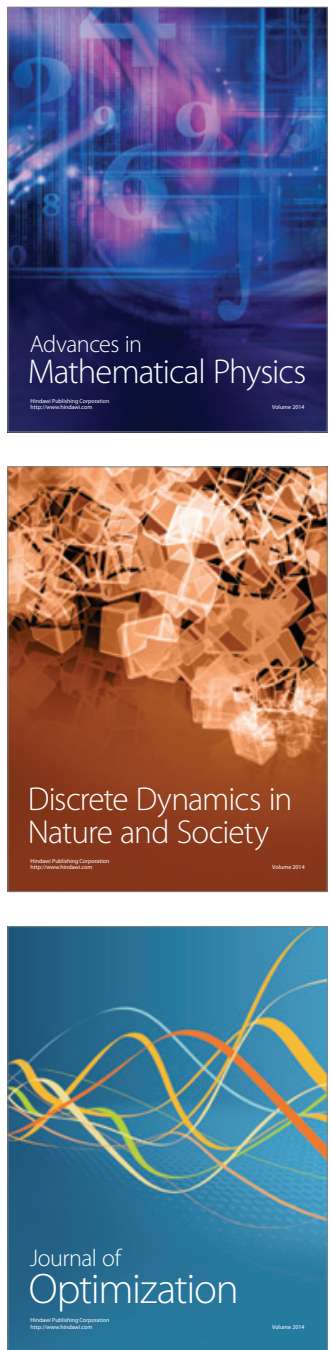